\title{
Two-loop cusp anomaly in ABJM at strong coupling
}

\author{
Lorenzo Bianchi, ${ }^{a}$ Marco S. Bianchi, ${ }^{a}$ Alexis Brès, ${ }^{a, b}$ Valentina Forini ${ }^{a}$ and \\ Edoardo Vescovi ${ }^{a}$ \\ ${ }^{a}$ Institut für Physik, Humboldt-Universität zu Berlin, \\ Newtonstraße 15, 12489 Berlin, Germany \\ ${ }^{b}$ Master ICFP, Département de Physique, École Normale Supérieure, \\ 24 rue Lhomond, 75231 Paris, France \\ E-mail: lorenzo.bianchi@physik.hu-berlin.de, \\ marco.bianchi@physik.hu-berlin.de, alexis.bres@ens.fr, \\ valentina.forini@physik.hu-berlin.de, \\ edoardo.vescovi@physik.hu-berlin.de
}

ABSTRACT: We compute the null cusp anomalous dimension of ABJM theory at strong coupling up to two-loop order. This is done by evaluating corrections to the corresponding superstring partition function, weighted by the $A d S_{4} \times \mathbb{C P}^{3}$ action in AdS light-cone gauge. We compare our result, where we use an anomalous shift in the $A d S_{4}$ radius, with the cusp anomaly of $\mathcal{N}=4 \mathrm{SYM}$, and extract the two-loop contribution to the non-trivial integrable coupling $h(\lambda)$ of ABJM theory. It coincides with the strong coupling expansion of the exact expression for $h(\lambda)$ recently conjectured by Gromov and Sizov. Our work provides thus a non-trivial perturbative check for the latter, as well as evidence for two-loop UV-finiteness and quantum integrability of the Type IIA $A d S_{4} \times \mathbb{C P}^{3}$ superstring in this gauge.

Keywords: AdS-CFT Correspondence, Chern-Simons Theories, Integrable Field Theories, Sigma Models

ARXIV EPRINT: 1407.4788 


\section{Contents}

1 Overview and results 1

2 AdS light-cone gauge in $A d S_{4} \times \mathbb{C P}^{3} \quad 6$

$\begin{array}{lll}3 & \text { The null cusp fluctuation action } & 9\end{array}$

$\begin{array}{lll}4 & \text { Cusp anomaly at one loop } & 10\end{array}$

5 Cusp anomaly at two loops 11

$\begin{array}{lll}5.1 \text { Bosonic sector } & 12\end{array}$

$\begin{array}{lll}5.2 & \text { Fermionic contributions } & 14\end{array}$

$\begin{array}{ll}5.3 \text { The cusp anomalous dimension } & 16\end{array}$

$\begin{array}{ll}5.4 \text { Comparison with } A d S_{5} \times S^{5} & 17\end{array}$

6 Concluding remarks $\quad 18$

$\begin{array}{ll}\text { A Lagrangian in the Wess-Zumino type parametrization } & 19\end{array}$

$\begin{array}{ll}\text { B Details on the expanded Lagrangian } & 21\end{array}$

$\begin{array}{ll}\text { C Integral reductions } & 22\end{array}$

\section{Overview and results}

A powerful attribute that the planar $\mathrm{AdS}_{4} / \mathrm{CFT}_{3}$ system [1] shares with its higherdimensional version, planar $\mathrm{AdS}_{5} / \mathrm{CFT}_{4}$ [2], is the conjectured integrability [3-6] of the gauge and (free) string theory model that define it - respectively $\mathcal{N}=6$ super ChernSimons-matter (ABJM) theory in $d=3$ and Type IIA superstrings in a $A d S_{4} \times \mathbb{C P}^{3}$ background with two- and four-form RR fluxes. The explicit realization of the integrable structure is however non-trivial, due to significative peculiarites of this case. A first one is the absence of maximal supersymmetry in the $A d S_{4} \times \mathbb{C P}^{3}$ background. This makes the construction of the corresponding superstring action difficult, in particular with issues on the $\kappa$-symmetry gauge-fixing suitable to describe strings moving only in AdS, the latter being a relevant setting for the studies of quantum integrability [7-9].

A second, crucial, peculiarity of the $\mathrm{AdS}_{4} / \mathrm{CFT}_{3}$ system is that all integrability-based calculations are given in terms of a non-trivial, interpolating function of the 't Hooft cou- 
pling $h(\lambda)$, appearing in the ABJM magnon dispersion relation ${ }^{1}$

$$
\epsilon=\frac{1}{2} \sqrt{1+16 h^{2}(\lambda) \sin ^{2} \frac{p}{2}}
$$

Its knowledge is decisive to grant the conjectured integrability of ABJM theory a full predictive power.

The first few orders of its weak coupling expansion were computed in [16-18] and in [19-21]. At strong coupling, one way to obtain information on $h(\lambda)$ is to evaluate in string theory the universal scaling function ${ }^{2}$ for the $\operatorname{ABJM}$ theory $f_{\mathrm{ABJM}}(\lambda)$, and then compare the result obtained with the asymptotic Bethe ansatz prediction of [4]. The latter is based on the equivalence of the BES [24] equations for the $\mathcal{N}=4$ case and the ABJM case and reads

$$
f_{\mathrm{ABJM}}(\lambda)=\left.\frac{1}{2} f_{\mathcal{N}=4}\left(\lambda_{\mathrm{YM}}\right)\right|_{\frac{\sqrt{\lambda_{\mathrm{YM}}}}{4 \pi} \rightarrow h(\lambda)},
$$

which implies

$$
f_{\mathrm{ABJM}}(\lambda)=2 h(\lambda)-\frac{3 \log 2}{2 \pi}-\frac{K}{8 \pi^{2}} \frac{1}{h(\lambda)}+\cdots,
$$

where $f_{\mathcal{N}=4}\left(\lambda_{\mathrm{YM}}\right)$ is the cusp anomaly of $\mathcal{N}=4 \mathrm{SYM}$ and $K$ is the Catalan constant. The leading strong coupling value for $f(\lambda)$ has been given already in [1] and reads $f(\lambda \gg 1)$ $=\sqrt{2 \lambda}$, from which via $(1.3)$ one gets $h(\lambda \gg 1)=\sqrt{\lambda / 2}$. At one loop in sigma-model perturbation theory, the scaling function has been evaluated in [25-37] via the energy of closed spinning strings in the large spin limit or similar means, providing a first subleading correction $-\log 2 /(2 \pi)$ to $h(\lambda)$ on which some debate existed [38]. In these calculations no issues were encountered in the action to use, as at one-loop only the quadratic part of the fermion Lagrangian is necessary, with a structure which is well-known in terms of the type IIA covariant derivative restricted by the background RR fluxes. ${ }^{3}$

We extend here the evaluation of the ABJM cusp anomaly to the two-loop order in sigma-model perturbation theory using the open string approach $[9,23]$ (in Type IIA), namely expanding the string partition function for the Euclidean surface ending on a null cusp at the boundary of $A d S_{4}$, as done in the $A d S_{5} \times S^{5}$ setting in [42]. As the classical string lies solely in $A d S_{4}$ and higher-order fermions are needed we must first face the problem, mentioned above, of using the correct superstring action. The coset $\operatorname{OSp}(6 \mid 4) /(\mathrm{U}(3) \times \mathrm{SO}(1,3))$ sigma-model formulation of it $[39,40]$ is built following the lines of (flat space and) type IIB superstrings [43], and exhibits classical integrability.

\footnotetext{
${ }^{1}$ In the $\mathcal{N}=4 \mathrm{SYM}$ case the relation of $h\left(\lambda_{\mathrm{YM}}\right)$ with the coupling is trivial at all orders, $h\left(\lambda_{\mathrm{YM}}\right)=$ $\sqrt{\lambda_{\mathrm{YM}}} /(4 \pi)$, as shown in [10-12] by evaluating the so-called "Brehmstrahlung function" both via an extrapolation on results of supersymmetric localization and via integrability. See also discussions in [13-15].

${ }^{2}$ Scaling function and cusp anomaly appear often as synonyms in the literature. At weak coupling and in the $\mathcal{N}=4$ case the scaling function $f\left(\lambda_{\mathrm{YM}}\right)$, multiplying the $\log S$ in the large spin anomalous dimensions of twist-two operators, equals twice the cusp anomalous dimension $\Gamma_{\text {cusp }}$ of light-like Wilson loops [22]. The same has been seen at strong coupling in [9, 23].

${ }^{3}$ Alternatively, one could still use the coset action of $[39,40]$ - which is not suitable when strings move confined in AdS $[40,41]$ — starting with a classical solution spinning both in $A d S_{4}$ with spin $S$ and in $\mathbb{C P}^{3}$ with spin $J$, and taking on the resulting expression for the one-loop energy a smooth $J \rightarrow 0$ limit [26].
} 
It can be interpreted as a partially gauge-fixed type IIA Green Schwarz action, where the $\kappa$-symmetry gauge-fixing sets to zero eight fermionic modes corresponding to the eight broken supersymmetries. However, as first argued in [40] and later clarified in [41], it is not suitable to describe the dynamics of a string lying solely in the $A d S_{4}$ part $^{4}$ of the $A d S_{4} \times \mathbb{C P}^{3}$ superspace, in that in this case four of the eight modes set to zero are in fact dynamical fermionic degrees of freedom of the superstring. Any action willing to capture the semiclassical dynamics on these classical string configurations should contain these physical fermions, and should therefore be found via another, sensible $\kappa$-symmetry gauge-fixing of the full action. This has been done in $[45,46]^{5}$, starting from the $D=11$ membrane action [48] based on the supercoset $\mathrm{OSp}(8 \mid 4) /(\mathrm{SO}(7) \times \mathrm{SO}(1,3))$, performing double dimensional reduction and choosing a $\kappa$-symmetry light-cone gauge for which both light-like directions lie in $A d S_{4}$. The output is an action, at most quartic in the fermions, which is the $A d S_{4} \times \mathbb{C P}^{3}$ counterpart of the gauge-fixed action of $[49,50]$. As the latter was efficiently used in [42] to evaluate the strong coupling corrections to the $\mathcal{N}=4 \mathrm{SYM}$ cusp anomaly up to two-loop order, the analysis of $[45,46]$ is the natural setup where to perform our calculation.

Any known classical string solution found in $A d S_{5}$, which can be embedded within an $A d S_{4}$ subspace, is immediately a solution for this theory [1]. Therefore we start using the null cusp solution of $[9,42]$ in the $A d S_{4} \times \mathbb{C P}^{3}$ action of $[45,46]$ and proceed evaluating corrections to the string path integral on it. These quantum string corrections are in general non-trivial to calculate, in connection with issues of potential UV divergences and the lack of manifest power-counting renormalizability of the string action when expanded around a particular background (see discussion in $[42,51-53]),{ }^{6}$ but have the additional important role of establishing the quantum consistency of the proposed string actions. This is a further motivation for the study at the quantum level of the action proposed in [45], where the more complicated structure of the $\mathbb{C P}^{3}$ background translates in a considerably more involved expression with respect to $[49,50]$. About the integrability of this string non-coset model, the standard analysis of [57] — which applies to the action of [40] is not possible here. The classical integrability of strings generically moving in the full $A d S_{4} \times \mathbb{C P}^{3}$ superspace has been however shown by constructing a Lax connection with zero curvature up to quadratic order in the fermions [58]. ${ }^{7}$

Similarly to the $A d S_{5} \times S^{5}$ case, the AdS light-cone approach to the evaluation of the cusp anomaly turns out to be extremely efficient. The background solution is "homogeneous", namely the fluctuation Lagrangian turns out to have only constant coefficients. This makes immediate the study of the fluctuation spectrum and highly simplifies the

\footnotetext{
${ }^{4}$ The same is true when the string forms a worldsheet instanton by wrapping a $\mathbb{C P}^{1}$ cycle in $\mathbb{C P}^{3}[44]$.

${ }^{5}$ See also [47].

${ }^{6}$ In the evaluation of the worldsheet S-matrix starting from the light-cone gauge fixed $A d S_{5} \times S^{5}$ GS superstring action, non-cancellation of UV divergences has been observed already beyond the tree-level order (see discussion in [54]). These issues, non present in alternative perturbative methods based on unitarity cuts [54-56], are still calling for an explanation.

${ }^{7} \mathrm{~A}$ study of classical integrability (prior to gauge-fixing) for general motion of the string in several backgrounds of interest for the AdS/CFT correspondence is in [59].
} 
semiclassical analysis at higher orders. ${ }^{8}$ Additional simplifications come from the fact that bosonic propagators in the AdS light-cone gauge are only diagonal, which limitates the number of Feynman graphs to be considered. ${ }^{9}$ In general, the actual calculation inherits from its $A d S_{5} \times S^{5}$ a similar mechanism of cancellation of divergences, and even the significative difference given by the presence of massless fermions in the spectrum turns out not to play a role (apart from the cancellation of UV divergences) in our final result, as they behave like effectively decoupled. The relevant interaction vertices are the same and no genuinely new contributions, in terms of scalar integrals, appears. This results in a different weight factor in front of the same structures ( $\log 2$ at one loop and the Catalan constant $K$ at two loops) appearing in the $A d S_{5} \times S^{5}$ case, where the weight is in terms of the ratio of the $A d S_{4}$ and $\mathbb{C P}^{3}$ radii, as well as the number of bosonic transverse AdS directions and massive fermions.

An important further ingredient in the $A d S_{4} \times \mathbb{C P}^{3}$ calculation is the correction to the effective string tension [64] which must be considered for the first time at this order in sigma-model perturbation theory. The original "dictionary" proposal [1] for the effective string tension in terms of the effective 't Hooft coupling $\lambda$ of ABJM reads

$$
T=\frac{R^{2}}{2 \pi \alpha^{\prime}}=2 \sqrt{2 \lambda}, \quad \lambda=\frac{N}{k},
$$

where $R$ is the $\mathbb{C P}^{3}$ radius. As pointed out in [64], the geometry (and flux, in the ABJ [65] theory) of the background induces higher order corrections to the radius of curvature in the Type IIA description, which in the planar limit of interest here appear in the form of a shift in the square root

$$
T=2 \sqrt{2\left(\lambda-\frac{1}{24}\right)} .
$$

We emphasize that the string perturbative expansion is an expansion in inverse string tension whose coefficients are obviously not affected by the correction (1.5). The radius shift is a (corrected) $\mathrm{AdS}_{4} / \mathrm{CFT}_{3}$ dictionary proposal, an assumed, new input which plays a role when expressing the result in terms of the 't Hooft coupling.

All this leads to the main result of this work, which is the evaluation of the first two strong coupling corrections to the ABJM cusp anomalous dimension

$$
f_{\mathrm{ABJM}}(\lambda)=\sqrt{2 \lambda}-\frac{5 \log 2}{2 \pi}-\left(\frac{K}{4 \pi^{2}}+\frac{1}{24}\right) \frac{1}{\sqrt{2 \lambda}}+\mathcal{O}(\sqrt{\lambda})^{-2}
$$

The formula can be rewritten in a more compact way defining the shifted coupling

$$
\tilde{\lambda} \equiv \lambda-\frac{1}{24}
$$

\footnotetext{
${ }^{8}$ The evaluation of perturbative (sigma-model) string corrections for non-homogenous solutions is currently limited to one-loop order, as in these cases in the fluctuation spectrum (and thus in the propagator) non-trivial special elliptic functions appear [37, 60-62] which depend on the worldsheet coordinates.

${ }^{9}$ In the first two-loop calculation of [63] the conformal gauge was used, in which propagators are nondiagonal, implying the evaluation of a larger number of two-loop diagrams.
} 
from which

$$
f_{\mathrm{ABJM}}(\tilde{\lambda})=\sqrt{2 \tilde{\lambda}}-\frac{5 \log 2}{2 \pi}-\frac{K}{4 \pi^{2} \sqrt{2 \tilde{\lambda}}}+\mathcal{O}(\sqrt{\tilde{\lambda}})^{-2}
$$

This form of the result makes evident the striking similarity with the $A d S_{5} \times S^{5}$ result

$$
f_{\mathrm{YM}}\left(\lambda_{\mathrm{YM}}\right)=\frac{\sqrt{\lambda_{\mathrm{YM}}}}{\pi}-\frac{3 \log 2}{\pi}-\frac{K}{\pi \sqrt{\lambda_{\mathrm{YM}}}}+\mathcal{O}\left(\sqrt{\lambda_{\mathrm{YM}}}\right)^{-2}
$$

where the change in the transcendentality pattern is due to the corresponding difference in the effective string tensions.

From (1.6) and via (1.2) we get then the strong-coupling two-loop correction for the interpolating function $h(\lambda)$, that we report here together with the weak coupling results $[16-21]$

$$
\begin{array}{ll}
h^{2}(\lambda)=\lambda^{2}-\frac{2 \pi^{3}}{3} \lambda^{4}+\mathcal{O}\left(\lambda^{6}\right) & \lambda \ll 1, \\
h(\lambda)=\sqrt{\frac{\lambda}{2}}-\frac{\log 2}{2 \pi}-\frac{1}{48 \sqrt{2 \lambda}}+\mathcal{O}(\sqrt{\lambda})^{-2} & \lambda \gg 1
\end{array}
$$

where we emphasize the a priori non-obvious fact the two-loop coefficient at strong coupling is only due to the anomalous radius shift.

A conjecture for the exact expression of $h(\lambda)$ has been recently made [66], in a spirit quite close to the one followed in $[10,11]$ on the comparison between two exact computations of the same observable (see footnote 1). The authors of [66] elaborated on the similarity between two all-order calculations in ABJM theory: one - the "slope function" [67] — derived via integrability as exact solution of a quantum spectral curve [6] and one - a 1/6 BPS Wilson loop [68-70] — obtained with supersymmetric localization. As the first of the two exact results is expressed in terms of the effective coupling $h(\lambda)$, an "extrapolation" for the latter has been derived in an exact, implicit, form. ${ }^{10}$ It is

$$
\lambda=\frac{\sinh 2 \pi h(\lambda)}{2 \pi}{ }_{3} F_{2}\left(\frac{1}{2}, \frac{1}{2}, \frac{1}{2} ; 1, \frac{3}{2} ;-\sinh ^{2} 2 \pi h(\lambda)\right),
$$

with weak and strong coupling expansions

$$
\begin{array}{ll}
h(\lambda)=\lambda-\frac{\pi^{2}}{3} \lambda^{3}+\frac{5 \pi^{4}}{12} \lambda^{5}-\frac{893 \pi^{6}}{1260} \lambda^{7}+\mathcal{O}\left(\lambda^{9}\right) & \lambda \ll 1, \\
h(\lambda)=\sqrt{\frac{1}{2}\left(\lambda-\frac{1}{24}\right)}-\frac{\log 2}{2 \pi}+\mathcal{O}\left(e^{-2 \pi \sqrt{2 \lambda}}\right) & \lambda \gg 1 .
\end{array}
$$

We see that (1.13) above, expanded for large $\lambda$, agrees with (1.10).

In general, the mutual consistency of several ingredients - our direct perturbative string calculation, the corrected dictionary of [64], the prediction (1.2)-(1.3) from the Bethe Ansatz [4] and the conjecture of [66] for the interpolating function $h(\lambda)$ - provides

\footnotetext{
${ }^{10}$ As noticed in [66], a more solid derivation of $h(\lambda)$ would require comparison between the localization results of $[69,70]$ and the ABJM Bremsstrahlung function [71-74], similarly to the case of the $h\left(\lambda_{\mathrm{YM}}\right)$ of $\mathcal{N}=4$ SYM, see footnote 1 .
} 
highly non-trivial evidence in support of the proposal (1.11) for the interpolating function $h(\lambda)$ of ABJM theory, and furnishes an indirect check of the quantum integrability of the $A d S_{4} \times \mathbb{C P}^{3}$ superstring theory in this $\kappa$-symmetry light-cone gauge.

The paper proceeds as follows. In section 2 we introduce the AdS light-cone gaugefixed action which in section 3 we write in terms of fluctuations over the null cusp classical solution. In section 4 we compute the one-loop correction to the cusp anomaly. In section 5 we extend the computation of the string partition function to one more order, verifying the cancellation of UV divergences and obtaining the strong coupling two-loop correction to the ABJM cusp anomaly. In appendix A we present for completeness a different parametrization of the $\kappa$-symmetry gauge-fixed action of [46] which can be transparently compared with its $A d S_{5} \times S^{5}$ counterpart. Appendices B and $\mathrm{C}$ contain, respectively, details on the expanded Lagrangian and explicit reductions for the relevant integrals which we use in section 5 .

\section{AdS light-cone gauge in $\operatorname{AdS}_{4} \times \mathbb{C P}^{3}$}

Our starting point is the $A d S_{4} \times \mathbb{C P}^{3}$ Lagrangian in the $\kappa$-symmetry light-cone gauge proposed in $[45,46]$. This is obtained by double dimensional reduction from the elevendimensional membrane action [48] based on the supercoset $\mathrm{OSp}(8 \mid 4) /(\mathrm{SO}(7) \times \mathrm{SO}(1,3))$, and choosing a $\kappa$-symmetry light-cone gauge for which both light-like directions lie in $A d S_{4}$. In the spirit of $[49,50]$ (and of earlier studies of brane models on the $A d S \times S$ backgrounds) the construction of $[45,46]$ formulates the bulk string theory in a way which is naturally related to the boundary CFT theory. In particular, the 32-dimensional spinors whose components are the coordinates associated to the odd generators of $\operatorname{OSp}(8 \mid 4)$ are divided in $\theta$ and $\eta$ fermions corresponding, respectively, to super-Poincaré generators and superconformal generators. The AdS $\kappa$-symmetry light-cone gauge consists in setting to zero that half of the fermions which correspond to fermionic generators having negative charge w.r.t. the $\mathrm{SO}(1,1)$ generator $M^{+-}$from the Lorentz group acting on the Minkowski boundary of $A d S_{4} \cdot{ }^{11}$ As our analysis below explicitly shows, it has the advantage of encompassing a quantum analysis of string configurations classically moving in the $A d S_{4}$ sector of $A d S_{4} \times \mathbb{C P}^{3} \cdot{ }^{12}$

The $A d S_{4} \times \mathbb{C P}^{3}$ background metric is

$$
d s_{10}^{2}=R^{2}\left(\frac{1}{4} d s_{A d S_{4}}^{2}+d s_{\mathbb{C P}^{3}}^{2}\right)
$$

where $R$ is the $\mathbb{C P}^{3}$ radius. For $A d S_{4}$ the Poincare patch is used and the parametrization of $\mathbb{C P}^{3}$ is at this stage arbitrary

$$
\begin{aligned}
d s_{A d S_{4}}^{2} & =\frac{d w^{2}+d x^{+} d x^{-}+d x^{1} d x^{1}}{w^{2}} & & x^{ \pm} \equiv x^{2} \pm x^{0}, \\
d s_{\mathbb{C P}^{3}}^{2} & =g_{M N} d z^{M} d z^{N} & & M=1, \ldots, 6 .
\end{aligned}
$$

\footnotetext{
${ }^{11}$ Another $\kappa$-symmetry gauge condition based on a similar "superconformal" basis has been considered in $[75]$.

${ }^{12} \mathrm{An}$ alternative $\kappa$-symmetry gauge fixing of the complete $A d S_{4} \times \mathbb{C P}^{3}$ superspace [41] which is suitable for studying regions of the theory that are not reachable by the supercoset sigma model of [39, 40] (see Introduction) has been considered in [47].
} 
Above, $x^{ \pm}$are the light-cone coordinates, $x^{m}=\left(x^{0}, x^{1}, x^{2}\right)$ parametrize the threedimensional boundary of $A d S_{4}$ and $w \equiv e^{2 \varphi}$ is the radial coordinate. The $\kappa$-symmetry light-cone gauge-fixed Lagrangian of $[45,46]$ can be written as follows ${ }^{13}$

$$
\begin{aligned}
S= & -\frac{T}{2} \int d \tau d \sigma L \\
L= & \gamma^{i j}\left\{\frac{e^{-4 \varphi}}{4}\left(\partial_{i} x^{+} \partial_{j} x^{-}+\partial_{i} x^{1} \partial_{j} x^{1}\right)+\partial_{i} \varphi \partial_{j} \varphi+g_{M N} \partial_{i} z^{M} \partial_{j} z^{N}\right. \\
& \left.+e^{-4 \varphi}\left(\partial_{i} x^{+} \varpi_{j}+\partial_{i} x^{+} \partial_{j} z^{M} h_{M}+e^{-4 \varphi} B \partial_{i} x^{+} \partial_{j} x^{+}\right)\right\} \\
& -2 \varepsilon^{i j} e^{-4 \varphi}\left(\omega_{i} \partial_{j} x^{+}+e^{-2 \varphi} C \partial_{i} x^{1} \partial_{j} x^{+}+\partial_{i} x^{+} \partial_{j} z^{M} \ell_{M}\right),
\end{aligned}
$$

where the string tension $T$ has been defined in (1.5) and the following quantities

$$
\begin{aligned}
\varpi_{i} & =i\left(\partial_{i} \theta_{a} \bar{\theta}^{a}-\theta_{a} \partial_{i} \bar{\theta}^{a}+\partial_{i} \theta_{4} \bar{\theta}^{4}-\theta_{4} \partial_{i} \bar{\theta}^{4}+\partial_{i} \eta_{a} \bar{\eta}^{a}-\eta_{a} \partial_{i} \bar{\eta}^{a}+\partial_{i} \eta_{4} \bar{\eta}^{4}-\eta_{4} \partial_{i} \bar{\eta}^{4}\right) \\
\omega_{i} & =\hat{\eta}_{a} \hat{\partial}_{i} \bar{\theta}^{a}+\hat{\partial}_{i} \theta_{a} \hat{\bar{\eta}}^{a}+\frac{1}{2}\left(\partial_{i} \theta_{4} \bar{\eta}^{4}-\partial_{i} \eta_{4} \bar{\theta}^{4}+\eta_{4} \partial_{i} \bar{\theta}^{4}-\theta_{4} \partial_{i} \bar{\eta}^{4}\right) \\
B & =8\left[\left(\hat{\eta}_{a} \hat{\bar{\eta}}^{a}\right)^{2}+\varepsilon_{a b c} \hat{\bar{\eta}}^{a} \hat{\bar{\eta}}^{b} \hat{\bar{\eta}}^{c} \bar{\eta}^{4}+\varepsilon^{a b c} \hat{\eta}_{a} \hat{\eta}_{b} \hat{\eta}_{c} \eta_{4}+2 \eta_{4} \bar{\eta}^{4}\left(\hat{\eta}_{a} \hat{\bar{\eta}}^{a}-\theta_{4} \bar{\theta}^{4}\right)\right] \\
C & =2 \hat{\eta}_{a} \hat{\bar{\eta}}^{a}+\theta_{4} \bar{\theta}^{4}+\eta_{4} \bar{\eta}^{4} \\
h_{M} & =2\left[\Omega_{M}^{a} \varepsilon_{a b c} \hat{\bar{\eta}}^{b} \hat{\bar{\eta}}^{c}-\Omega_{a M} \varepsilon^{a b c} \hat{\eta}_{b} \hat{\eta}_{c}+2\left(\Omega_{a M} \hat{\bar{\eta}}^{a} \bar{\eta}^{4}-\Omega_{M}^{a} \hat{\eta}_{a} \eta_{4}\right)+2\left(\theta_{4} \bar{\theta}^{4}+\eta_{4} \bar{\eta}^{4}\right) \tilde{\Omega}_{a M}^{a}\right] \\
\ell_{M} & =2 i\left[\Omega_{a M} \hat{\bar{\eta}}^{a} \bar{\theta}^{4}+\Omega_{M}^{a} \hat{\eta}_{a} \theta_{4}+\left(\theta_{4} \bar{\eta}^{4}-\eta_{4} \bar{\theta}^{4}\right) \tilde{\Omega}_{a M}^{a}\right]
\end{aligned}
$$

include fermions up to the fourth power. As in the $A d S_{5} \times S^{5}$ case [49, 50], the action is quadratic in the $\theta$-fermions and quartic in the $\eta$-fermions.

Above, the fermionic coordinates $\eta_{a}$ and $\theta_{a}$ (and their conjugates) transform in the fundamental (antifundamental) representation of $\mathrm{SU}(3)(a=1,2,3)$, and correspond to the unbroken 24 supersymmetries of the $A d S_{4} \times \mathbb{C P}^{3}$ background. The remaining fermions $\eta_{4}, \theta_{4}$ and their conjugates originate from the eight broken supersymmetries. The manifest symmetry of the action is thus only the $\mathrm{SU}(3)$ subgroup of the $\mathrm{SU}(4)$ global symmetry of $\mathbb{C P}^{3}$. This feature, as we will see, will be inherited by the quantum fluctuations around the light-like cusp (see also discussion in appendix A). The $\Omega_{M}^{a}$ and $\Omega_{a M}$ appearing in the Lagrangian are the complex vielbein of $\mathbb{C P}^{3}, d s_{\mathbb{C P}^{3}}^{2}=\Omega_{M}^{a} \Omega_{a N} d z^{M} d z^{N}$, namely components of the Cartan one-forms of SU(4)/U(3), $\Omega^{a}=\Omega_{M}^{a} d z^{M}$ and $\Omega_{a}=\Omega_{a M} d z^{M}$. In the construction of [45], $\tilde{\Omega}_{a}{ }^{a}$ is associated to a one-form corresponding to the fiber direction of $S^{7}$. Its expression is given explicitly below in terms of the $\mathbb{C P}^{3}$ coordinates. The $\Omega_{M}^{a}$ and $\tilde{\Omega}_{a}^{a}$ appear in [45] in a "dressed" $\operatorname{OSp}(6 \mid 4) /(\mathrm{U}(3) \times \mathrm{SO}(1,3))$ supercoset element where the dressing incorporates the information on the broken supersymmetries and $U(1)$ fiber

\footnotetext{
${ }^{13}$ Inspired by [49] we modify the action proposed in $[45,46]$ with a convenient rescaling of the fermions

$$
\theta_{a} \rightarrow \sqrt{2} \theta_{a} \quad \theta_{4} \rightarrow \sqrt{2} e^{-\varphi} \theta_{4} \quad \eta_{a} \rightarrow \sqrt{2} e^{-2 \varphi} \eta_{a} \quad \eta_{4} \rightarrow \sqrt{2} e^{-\varphi} \eta_{4}
$$

and similar ones for the complex conjugates. With respect to $[45,46]$, we also partially change notation. 
direction. In (2.6), hatted quantities are related to unhatted ones via a rotation by matrices $T$ (similar matrices were conveniently introduced in [50]) which depend on the $\mathbb{C P}^{3}$ coordinates and act as follows on e.g. a $\eta_{a}$ fermion

$$
\hat{\eta}_{a}=T_{a}{ }^{b} \eta_{b}+T_{a b} \bar{\eta}^{b}, \quad \hat{\bar{\eta}}^{a}=T_{b}^{a} \bar{\eta}^{b}+T^{a b} \eta_{b} .
$$

In appendix A we rewrite the Lagrangian (2.5) in a form that is more similar to the $A d S_{5} \times S^{5}$ of [49], and comment more on the Cartan forms $\Omega$ and $T$-matrices.

The parametrization for $\mathbb{C P}^{3}$ chosen in [76] consists of complex variables $z^{a}$ and $\bar{z}_{a}$, transforming in the $\mathbf{3}$ and $\overline{\mathbf{3}}$ of SU(3) respectively. Then the metric reads

$$
d s_{\mathbb{C P}^{3}}^{2}=g_{a b} d z^{a} d z^{b}+g^{a b} d \bar{z}_{a} d \bar{z}_{b}+2 g_{a}^{b} d z^{a} d \bar{z}_{b},
$$

where

$$
\begin{aligned}
& g_{a b}=\frac{1}{4|z|^{4}}\left(|z|^{2}-\sin ^{2}|z|+\sin ^{4}|z|\right) \bar{z}_{a} \bar{z}_{b}, \quad g^{a b}=\frac{1}{4|z|^{4}}\left(|z|^{2}-\sin ^{2}|z|+\sin ^{4}|z|\right) z^{a} z^{b}, \\
& g_{a}{ }^{b}=\frac{\sin ^{2}|z|}{2|z|^{2}} \delta_{a}^{b}+\frac{1}{4|z|^{4}}\left(|z|^{2}-\sin ^{2}|z|-\sin ^{4}|z|\right) \bar{z}_{a} z^{b} \quad \text { and } \quad|z|^{2} \equiv z^{a} \bar{z}_{a} .
\end{aligned}
$$

For the one-forms appearing in the Lagrangian explicit expressions then follow, which can be derived from their definition

$$
\Omega^{a}=\Omega_{, b}^{a} d z^{b}+\Omega^{a, b} d \bar{z}_{b}, \quad \Omega_{a}=\Omega_{a, b} d z^{b}+\Omega_{a}{ }^{b} d \bar{z}_{b}, \quad \tilde{\Omega}_{a}^{a}=\tilde{\Omega}_{a, b}^{a} d z^{b}+\tilde{\Omega}_{a}^{a, b} d \bar{z}_{b}
$$

using (A.7). For example,

$$
\tilde{\Omega}_{a}^{a}=i \frac{\sin ^{2}|z|}{|z|^{2}}\left(d z^{a} \bar{z}_{a}-z^{a} d \bar{z}_{a}\right)
$$

In this parametrization, the T-matrices introduced in (2.12) can be grouped in a unitary matrix $T_{\hat{a}}^{\hat{b}}$ which reads explicitly [76]

$$
T_{\hat{a}}^{\hat{b}}=\left(\begin{array}{cc}
T_{a}^{b} & T_{a b} \\
T^{a b} & T_{b}^{a}
\end{array}\right)=\left(\begin{array}{cc}
\delta_{a}^{b} \cos |z|+\bar{z}_{a} z^{b} \frac{1-\cos |z|}{|z|^{2}} & i \varepsilon_{a c b} z^{c} \frac{\sin |z|}{|z|} \\
-i \varepsilon^{a c b} \bar{z}_{c} \frac{\sin |z|^{\mid}}{|z|} & \delta_{b}^{a} \cos |z|+z^{a} \bar{z}_{b} \frac{1-\cos |z|}{|z|^{2}}
\end{array}\right) .
$$

The action (2.5) has gauge-fixed local fermionic symmetry. To fix bosonic local symmetry and further proceed with our analysis it is convenient to use, as discussed in [49] and used in $[42,52,53]$, a "modified" conformal gauge

$$
\gamma^{i j}=\operatorname{diag}\left(-e^{4 \varphi}, e^{-4 \varphi}\right),
$$

in combination with the standard light-cone gauge

$$
x^{+}=p^{+} \tau, \quad p^{+}=\text {const } .
$$

In what follows we will give directly the expression of the Euclidean version of the action (2.5) in this gauge (choosing $p^{+}=1$ ) and on the null cusp background [9, 42]. 


\section{The null cusp fluctuation action}

In this section we consider the Wick-rotated, Euclidean formulation of the Lagrangian (2.5) in the bosonic light-cone gauge (2.18)-(2.19) and compute its fluctuations about the null cusp background. The equations of motion derived from the (Euclidean) AdS light-cone gauge Lagrangian (2.5) admit a classical solution for which the on-shell action is the area of the minimal surface ending on a null cusp on the $A d S_{4}$ boundary. This configuration is just the $A d S_{4}$ embedding of the classical string solution found in the $A d S_{5}$ background [9, 42], and reads

$$
\begin{aligned}
& w \equiv e^{2 \varphi}=\sqrt{\frac{\tau}{\sigma}} \quad x^{1}=0 \\
& x^{+}=\tau \quad x^{-}=-\frac{1}{2 \sigma} \quad z^{M}=0 .
\end{aligned}
$$

The requirement that the open string Euclidean world-sheet described by these coordinates ends on a cusp at the boundary of $A d S_{4}$ at $w=0$ is manifestly enforced by the relation $x^{+} x^{-}=-\frac{1}{2} w^{2}$. In the AdS/CFT dictionary of [7, 77], the Wilson loop evaluated on a light-like cusp contour is then given by the superstring partition function

$$
\left\langle W_{\text {cusp }}\right\rangle=Z_{\text {string }} \equiv \int \mathcal{D}[x, w, z, \eta, \theta] e^{-S_{E}} .
$$

In order to compute it perturbatively, we first construct the Euclidean action $S_{E}$ for fluctuations about the background (3.1). Following [42], we will use a suitable parametrization of fluctuations which, combined with a further redefinition of the worldsheet coordinates $t=\log \tau$ and $s=\log \sigma$, is such that the coefficients of the fluctuation action become constant, namely $(\tau, \sigma)$-independent. It reads ${ }^{14}$

$$
\begin{aligned}
& x^{1}=2 \sqrt{\frac{\tau}{\sigma}} \tilde{x}^{1} \\
& w=\sqrt{\frac{\tau}{\sigma}} \tilde{w} \\
& \tilde{w}=e^{2 \tilde{\varphi}} \\
& z^{a}=\tilde{z}^{a} \\
& \bar{z}^{a}=\tilde{\bar{z}}^{a} \\
& a=1,2,3 \\
& \eta=\frac{1}{\sqrt{\sigma}} \tilde{\eta} \\
& \theta=\frac{1}{\sqrt{\sigma}} \tilde{\theta} .
\end{aligned}
$$

After the Wick rotation $\tau \rightarrow-i \tau, p^{+} \rightarrow i p^{+}$and having set $p^{+}=1$, we end up with the following action for fluctuations over the null-cusp background (3.1)

$$
S_{E}=\frac{T}{2} \int d t d s \mathcal{L}, \quad \mathcal{L}=\mathcal{L}_{B}+\mathcal{L}_{F}^{(2)}+\mathcal{L}_{F}^{(4)},
$$

where

$$
\begin{aligned}
\mathcal{L}_{B}= & \left(\partial_{t} \tilde{x}^{1}+\frac{1}{2} \tilde{x}^{1}\right)^{2}+\frac{1}{\tilde{w}^{4}}\left(\partial_{s} \tilde{x}^{1}-\frac{1}{2} \tilde{x}^{1}\right)^{2}+\tilde{w}^{2}\left(\partial_{t} \varphi\right)^{2}+\frac{1}{\tilde{w}^{2}}\left(\partial_{s} \varphi\right)^{2}+\frac{1}{16}\left(\tilde{w}^{2}+\frac{1}{\tilde{w}^{2}}\right)+ \\
& +\tilde{w}^{2} \tilde{g}_{M N} \partial_{t} \tilde{z}^{M} \partial_{t} \tilde{z}^{N}+\frac{1}{\tilde{w}^{2}} \tilde{g}_{M N} \partial_{s} \tilde{z}^{M} \partial_{s} \tilde{z}^{N}
\end{aligned}
$$

\footnotetext{
${ }^{14}$ The factor 2 in the fluctuation of the field $x^{1}$ is introduced to normalize the kinetic term of $\tilde{x}^{1}$.
} 


$$
\begin{aligned}
\mathcal{L}_{F}^{(2)}= & i\left[\partial_{t} \tilde{\theta}_{a} \tilde{\bar{\theta}}^{a}-\tilde{\theta}_{a} \partial_{t} \tilde{\bar{\theta}}^{a}+\partial_{t} \tilde{\theta}_{4} \tilde{\bar{\theta}}^{4}-\tilde{\theta}_{4} \partial_{t} \tilde{\bar{\theta}}^{4}+\partial_{t} \tilde{\eta}_{a} \tilde{\bar{\eta}}^{a}-\tilde{\eta}_{a} \partial_{t} \tilde{\bar{\eta}}^{a}+\partial_{t} \tilde{\eta}_{4} \tilde{\bar{\eta}}^{4}-\tilde{\eta}_{4} \partial_{t} \tilde{\bar{\eta}}^{4}\right]+ \\
& +\frac{2 i}{w^{2}}\left[\hat{\eta}_{a}\left(\hat{\partial}_{s} \bar{\theta}^{a}-\frac{1}{2} \hat{\bar{\theta}}^{a}\right)+\left(\hat{\partial}_{s} \theta_{a}-\frac{1}{2} \hat{\theta}_{a}\right) \hat{\bar{\eta}}^{a}+\frac{1}{2}\left(\partial_{s} \theta_{4} \bar{\eta}^{4}-\partial_{s} \eta_{4} \bar{\theta}^{4}+\eta_{4} \partial_{s} \bar{\theta}^{4}-\theta_{4} \partial_{s} \bar{\eta}^{4}\right)\right] \\
& +\partial_{t} \tilde{z}^{M} \tilde{h}_{M}+\frac{4 i}{\tilde{w}^{3}} \tilde{C}\left(\partial_{s} \tilde{x}^{1}-\frac{1}{2} \tilde{x}^{1}\right)-\frac{2 i}{\tilde{w}^{2}} \partial_{s} \tilde{z}^{M} \tilde{\ell}_{M} \\
\mathcal{L}_{F}^{(4)}= & \frac{1}{\tilde{w}^{4}} \tilde{B} .
\end{aligned}
$$

In the expressions above, with $\tilde{B}, \tilde{C}, \tilde{h}_{M}$ and $\tilde{\ell}_{M}$ we indicate the quantities $B, C, h_{M}$ and $\ell_{M}$ in (2.6) where a tilde over each field appears (namely, the weighting factors for the fluctuations in (3.3) have already been made explicit in the derivatives of products).

Since the Lagrangian has now constant coefficients and is thus translationally invariant, the (infinite) world-sheet volume factor $V$ factorizes. The scaling function is then defined via the string partition function as [42]

$$
W=-\ln Z=\frac{1}{2} f(\lambda) V=W_{0}+W_{1}+W_{2}+\ldots, \quad V=\frac{1}{4} V_{2} \equiv \frac{1}{4} \int d t d s
$$

where $W_{0} \equiv S_{E}$ coincides with the value of the action on the background, $W_{1}, W_{2}, \ldots$ are one-, two- and higher loop corrections, and for the ratio $V / V_{2}$ we use the same convention as in [42]. ${ }^{15}$ From (3.8) we explicitly define $f(\lambda)$ in terms of the effective action $W$

$$
f(\lambda)=\frac{8}{V_{2}} W
$$

We are now ready to compute the effective action perturbatively in inverse powers of the effective string tension $g \equiv \frac{T}{2}$. From this we will extract the corresponding strong coupling perturbative expansion for the scaling function

$$
f(g)=g\left[1+\frac{a_{1}}{g}+\frac{a_{2}}{g^{2}}+\ldots\right], \quad g=\frac{T}{2} .
$$

where we have factorized the classical result from $W_{0}=S_{E}[1]$ and the effective string tension $T$ is defined in (1.5).

\section{Cusp anomaly at one loop}

We start considering one-loop quantum corrections to the free energy (3.2), which are derived expanding the fluctuation Lagrangian (3.4) to second order in the fields.

For the bosonic part we obtain

$$
\mathcal{L}_{B}^{(2)}=\left(\partial_{t} \tilde{x}^{1}\right)^{2}+\left(\partial_{s} \tilde{x}^{1}\right)^{2}+\frac{1}{2}\left(\tilde{x}^{1}\right)^{2}+\left(\partial_{t} \tilde{\varphi}\right)^{2}+\left(\partial_{s} \tilde{\varphi}\right)^{2}+\tilde{\varphi}^{2}+\left|\partial_{t} \tilde{z}^{a}\right|^{2}+\left|\partial_{s} \tilde{z}^{a}\right|^{2} .
$$

The bosonic degrees of freedom consist of six real massless scalars (associated to the $\mathbb{C P}^{3}$ coordinates), one real scalar $\tilde{x}^{1}$ with mass $m^{2}=\frac{1}{2}$ and one real scalar $\tilde{\varphi}$ with mass $m^{2}=1$.

\footnotetext{
${ }^{15}$ This is related to coordinate transformation and field redefinitions occurring between the GKP [8] string, whose energy is given in terms of $f(\lambda)$, and the null cusp solution in the Poincaré patch here used, see discussion in [53].
} 
This is a simple truncation (one less transverse degree of freedom in the AdS space) of the bosonic spectrum found in the $A d S_{5} \times S^{5}$ [42]. For the fermions one gets an off-diagonal kinetic matrix

$$
\mathcal{L}_{F}^{(2)}=i \Theta K_{F} \Theta^{T} \quad \text { where } \quad \Theta \equiv\left(\tilde{\theta}_{a}, \tilde{\theta}_{4}, \tilde{\bar{\theta}}^{a}, \tilde{\bar{\theta}}^{4}, \tilde{\eta}_{a}, \tilde{\eta}_{4}, \tilde{\bar{\eta}}^{a}, \tilde{\bar{\eta}}^{4}\right)
$$

which reads

$$
K_{F}=\left(\begin{array}{cccccccc}
0 & 0 & -\partial_{t} & 0 & 0 & 0 & -\partial_{s}-\frac{1}{2} & 0 \\
0 & 0 & 0 & -\partial_{t} & 0 & 0 & 0 & -\partial_{s} \\
-\partial_{t} & 0 & 0 & 0 & \partial_{s}+\frac{1}{2} & 0 & 0 & 0 \\
0 & -\partial_{t} & 0 & 0 & 0 & \partial_{s} & 0 & 0 \\
0 & 0 & \partial_{s}-\frac{1}{2} & 0 & 0 & 0 & -\partial_{t} & 0 \\
0 & 0 & 0 & \partial_{s} & 0 & 0 & 0 & -\partial_{t} \\
-\partial_{s}+\frac{1}{2} & 0 & 0 & 0 & -\partial_{t} & 0 & 0 & 0 \\
0 & -\partial_{s} & 0 & 0 & 0 & -\partial_{t} & 0 & 0
\end{array}\right) .
$$

Fermions contribute to the partition function with the determinant $\left(\partial_{\mu}=i p_{\mu}, \mu=0,1\right)$

$$
\operatorname{det} K_{F}=\left(p^{2}\right)^{2}\left(p^{2}+\frac{1}{4}\right)^{6},
$$

from which we read that the fermionic spectrum is composed of six massive degrees of freedom with mass $m^{2}=1 / 4$ and two massless ones. The latter are of $\eta_{4}$ and $\theta_{4}$ type, namely those fermionic directions corresponding to the broken supersymmetries. The presence of massless fermions marks a difference with respect to the $\mathcal{N}=4$ SYM case, already noticed in this theory when studying fluctuations over classical string solutions only lying in $A d S_{4}[28,32,36,37]$ (see comments in section 5.4).

The one-loop effective action is computed as

$$
W_{1}=-\log Z_{1}
$$

where $Z_{1}$ is the ratio of fermionic over bosonic determinants. Therefore

$$
W_{1}=\frac{1}{2} V_{2} \int \frac{d^{2} p}{(2 \pi)^{2}}\left\{\log \left(p^{2}+1\right)+\log \left(p^{2}+\frac{1}{2}\right)+4 \log \left(p^{2}\right)-6 \log \left(p^{2}+\frac{1}{4}\right)\right\}=-\frac{5 \log 2}{16 \pi} V_{2} .
$$

The one-loop correction to the scaling function reads, according to (3.9),

$$
a_{1}=-\frac{5 \log 2}{2 \pi}
$$

and agrees with previous independent results $[28,32,36]$.

\section{Cusp anomaly at two loops}

In this section we provide the details on the computation of the two-loop coefficient of the scaling function. The calculation follows the lines of [42], with some important differences which we point out in section 5.4. In particular the aim is to compute the connected vacuum 

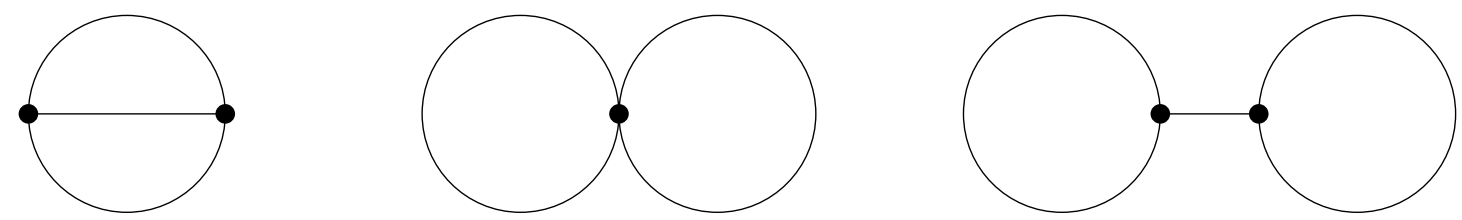

Figure 1. Sunset, double bubble and double tadpole are the diagrams appearing in the two-loop contribution to the partition function.

diagrams of the fluctuation Lagrangian around the null cusp background. Denoting by $W$ the free energy of the theory, $W=-\log Z$, the two-loop contribution is given by

$$
W_{2}=\left\langle S_{\text {int }}\right\rangle-\frac{1}{2}\left\langle S_{\text {int }}^{2}\right\rangle_{c},
$$

where $S_{\text {int }}$ is the interacting part of the action at cubic and quadratic order (see appendix B). The subscript $c$ indicates that only connected diagrams need to be included. In the following we use $S_{\text {int }}=T \int d t d s \mathcal{L}_{\text {int }}$ and we give the expressions of the vertices as they appear in $\mathcal{L}_{\text {int }}$. Throughout this section we drop tildes from fluctuation fields in order not to clutter formulae. Also, we neglect the string tension $T$ and the volume $V_{2}$ in the intermediate steps and reinstate them at the end of the calculation.

\subsection{Bosonic sector}

Let us first consider the purely bosonic sector. As pointed out in section 4, the spectrum of the theory contains one real boson of squared mass 1 , one real boson of squared mass $\frac{1}{2}$ and three complex massless bosons. The interaction among these excitations involves cubic and quartic vertices which give rise to the diagrams in figure 1.

We observe that the AdS light-cone gauge Lagrangian contains only diagonal bosonic propagators, which introduces considerable simplifications in the perturbative computation. The explicit expressions of the propagators are

$$
G_{\varphi \varphi}(p)=\frac{1}{p^{2}+1} \quad G_{z_{a} \bar{z}^{b}}(p)=\frac{2 \delta_{a}^{b}}{p^{2}} \quad G_{x^{1} x^{1}}(p)=\frac{1}{p^{2}+\frac{1}{2}} .
$$

The cubic interactions involving only bosonic fields are of three different kinds

$$
V_{\varphi x^{1} x^{1}}=-4 \varphi\left[\left(\partial_{s}-\frac{1}{2}\right) x^{1}\right]^{2} \quad V_{\varphi^{3}}=2 \varphi\left[\left(\partial_{t} \varphi\right)^{2}-\left(\partial_{s} \varphi\right)^{2}\right] \quad V_{\varphi|z|^{2}}=2 \varphi\left[\left|\partial_{t} z\right|^{2}-\left|\partial_{s} z\right|^{2}\right] .
$$

When combining vertices and propagators in the sunset diagrams they originate various non-covariant integrals with components of the loop momenta in the numerators. Standard reduction techniques allow to rewrite every integral as a linear combination of the two following scalar ones (explicit reductions for the relevant integrals are spelled out in appendix C)

$$
\begin{aligned}
I\left(m^{2}\right) & \equiv \int \frac{d^{2} p}{(2 \pi)^{2}} \frac{1}{p^{2}+m^{2}} \\
I\left(m_{1}^{2}, m_{2}^{2}, m_{3}^{2}\right) & \equiv \int \frac{d^{2} p d^{2} q d^{2} r}{(2 \pi)^{4}} \frac{\delta^{(2)}(p+q+r)}{\left(p^{2}+m_{1}^{2}\right)\left(q^{2}+m_{2}^{2}\right)\left(r^{2}+m_{3}^{2}\right)} .
\end{aligned}
$$


The latter integral is finite, provided none of the masses vanishes, and is otherwise IR divergent. The former is clearly UV logarithmically divergent, and also develops IR singularities in the massless case. In our computation we expect all UV divergences to cancel and therefore no divergent integral to appear in the final result. Nonetheless, performing reduction of potentially divergent tensor integrals to scalar ones still implies the choice of a regularization scheme. In our case we use the one adopted in $[42,51,78]$. This prescription consists of performing all manipulations in the numerators in $d=2$, which has the advantage of simpler tensor integral reductions. In this process we set to zero power UV divergent massless tadpoles, as in dimensional regularization

$$
\int \frac{d^{2} p}{(2 \pi)^{2}}\left(p^{2}\right)^{n}=0, \quad n \geq 0 .
$$

All remaining logarithmically divergent integrals happen to cancel out in the computation and there is no need to pick up an explicit regularization scheme to compute them.

As an explicit example, we consider the contribution to the sunset coming from the first vertex in (5.3)

$$
-\frac{1}{2}\left\langle V_{\varphi x^{1} x^{1}}^{2}\right\rangle=-\int \frac{d^{2} p d^{2} q d^{2} r}{(2 \pi)^{4}} \frac{\left(1+4 q_{1}^{2}\right)\left(1+4 r_{1}^{2}\right) \delta^{(2)}(p+q+r)}{\left(p^{2}+1\right)\left(q^{2}+\frac{1}{2}\right)\left(r^{2}+\frac{1}{2}\right)}=\frac{1}{2} I\left(1, \frac{1}{2}, \frac{1}{2}\right) .
$$

The reason why the coefficient of the integral in the second term of (5.7) is exactly $(-1)$ is the topic of section 5.4. We note that the integral $I\left(1, \frac{1}{2}, \frac{1}{2}\right)$ already appeared in [42] and is a particular case of the general class

$$
I\left(2 m^{2}, m^{2}, m^{2}\right)=\frac{K}{8 \pi^{2} m^{2}}
$$

where $K$ is the Catalan constant

$$
K \equiv \sum_{n=0}^{\infty} \frac{(-1)^{n}}{(2 n+1)^{2}}
$$

The contribution of the sunset diagram involving the second vertex in (5.3) is proportional to $I(1)^{2}$, whereas the contribution of the third vertex vanishes

$$
-\frac{1}{2}\left\langle V_{\varphi^{3}}^{2}\right\rangle=2 I(1)^{2} \quad-\frac{1}{2}\left\langle V_{\varphi|z|^{2}}^{2}\right\rangle=0
$$

The final contribution of the bosonic sunset diagrams is

$$
W_{2, \text { bos. sunset }}=\frac{1}{2} I\left(1, \frac{1}{2}, \frac{1}{2}\right)+2 I(1)^{2} .
$$

The first two vertices in (5.3) can also be contracted to generate non-1PI graphs, namely double tadpoles. However the resulting diagrams turn out to vanish individually. 
Next we consider bosonic double bubble diagrams. The relevant quartic vertices are

$$
\begin{aligned}
V_{\varphi^{2} x^{1} x^{1}}= & 16 \varphi^{2}\left[\left(\partial_{s}-\frac{1}{2}\right) x^{1}\right]^{2} \\
V_{\varphi^{4}}= & 4 \varphi^{2}\left[\left(\partial_{t} \varphi\right)^{2}+\left(\partial_{s} \varphi\right)^{2}+\frac{1}{6} \varphi^{2}\right] \\
V_{\varphi^{2}|z|^{2}}= & 4 \varphi^{2}\left[\left|\partial_{t} z\right|^{2}+\left|\partial_{s} z\right|^{2}\right] \\
V_{z^{4}}= & \frac{1}{6}\left[\left(\bar{z}_{a} \partial_{t} z^{a}\right)^{2}+\left(\bar{z}_{a} \partial_{s} z^{a}\right)^{2}+\left(z^{a} \partial_{t} \bar{z}_{a}\right)^{2}+\left(z^{a} \partial_{s} \bar{z}_{a}\right)^{2}\right. \\
& \left.-|z|^{2}\left(\left|\partial_{t} z\right|^{2}+\left|\partial_{s} z\right|^{2}\right)-\left|\bar{z}_{a} \partial_{t} z^{a}\right|^{2}-\left|\bar{z}_{a} \partial_{s} z^{a}\right|^{2}\right] .
\end{aligned}
$$

Despite the lengthy expressions of the vertices, the only non-vanishing contribution comes from $V_{\varphi^{4}}$ and gives

$$
W_{2, \text { bos. bubble }}=-2 I(1)^{2}
$$

and cancels the divergent part of (5.11). As a result, the bosonic sector turns out to be free of divergences without the need of fermonic contributions (as it happens at one loop), which was already observed in the $A d S_{5} \times S^{5}$ case [42].

\subsection{Fermionic contributions}

We compute the diagrams arising from interactions involving fermions. The fermionic propagators can be read from the inverse of the kinetic matrix $K_{F}(4.3)$

$$
\begin{aligned}
& G_{\eta_{4} \bar{\eta}^{4}}(p)=G_{\theta_{4} \bar{\theta}^{4}}(p)=\frac{p_{0}}{p^{2}} \quad G_{\eta_{4} \bar{\theta}^{4}}(p)=G_{\theta_{4} \bar{\eta}^{4}}(-p)=-\frac{p_{1}}{p^{2}} \\
& G_{\eta_{a} \bar{\eta}^{b}}(p)=G_{\theta_{a} \bar{\theta}^{b}}(p)=\frac{p_{0}}{p^{2}+\frac{1}{4}} \delta_{a}^{b} \quad G_{\eta_{a} \bar{\theta}^{b}}(p)=G_{\theta_{a} \bar{\eta}^{b}}(-p)=-\frac{p_{1}+\frac{i}{2}}{p^{2}+\frac{1}{4}} \delta_{a}^{b}
\end{aligned}
$$

The main difference between the spectrum of $A d S_{5} \times S^{5}$ and the one introduced in section 4 resides in the fermionic part. Although both theories have eight fermionic degrees of freedom, in $A d S_{4} \times \mathbb{C P}^{3}$ they are split into six massive and two massless excitations, which interact non-trivially among themselves.

We start by considering diagrams involving at least one massless fermion. The relevant cubic vertices are (we denote by $\psi$ the fermions $\eta$ and $\theta$ collectively)

$$
\begin{aligned}
V_{z \eta_{a} \eta_{4}} & =-2 \partial_{t} z^{a} \eta_{a} \eta_{4}+h . c . & V_{z \eta_{a} \theta_{4}} & =2 \partial_{s} z^{a} \eta_{a} \theta_{4}-h . c . \\
V_{\varphi \eta_{4} \bar{\theta}^{4}} & =-2 i \varphi\left(\bar{\theta}^{4} \partial_{s} \eta_{4}-\partial_{s} \bar{\theta}^{4} \eta_{4}\right)-h . c . & V_{x^{1} \bar{\psi}^{4} \psi_{4}} & =-2 i\left(\bar{\eta}^{4} \eta_{4}+\bar{\theta}^{4} \theta_{4}\right)\left(\partial_{s}-\frac{1}{2}\right) x^{1} .
\end{aligned}
$$

The quartic interactions are either not suitable for constructing a double tadpole diagram or they produce vanishing integrals. These include vector massless tadpoles, which vanish by parity, and tensor massless tadpoles, which have power UV divergences and are set to zero. For completeness we list them in appendix B.

Focussing on the Feynman graphs which can be constructed from cubic interaction we also note that the only double tadpole diagrams that can be produced using (5.18) involve tensor massless tadpole integrals and therefore vanish. In the sector with massless fermions 
we are therefore left with the sunset diagrams, which, thanks to the diagonal structure of the bosonic propagators, turn out to be only five

$$
W_{2, \psi_{4}}=-\frac{1}{2}\left\langle V_{z \eta_{a} \eta_{4}} V_{z \eta_{a} \eta_{4}}+V_{z \eta_{a} \theta_{4}} V_{z \eta_{a} \theta_{4}}+2 V_{z \eta_{a} \eta_{4}} V_{z \eta_{a} \theta_{4}}+V_{\varphi \eta_{4} \bar{\theta}^{4}} V_{\varphi \eta_{4} \bar{\theta}^{4}}+V_{x^{1} \bar{\psi}^{4} \psi_{4}} V_{x^{1} \bar{\psi}^{4} \psi_{4}}\right\rangle
$$

The explicit computation of the individual contributions shows that they are all vanishing. As an example we consider

$$
-\frac{1}{2}\left\langle V_{\varphi \eta_{4} \bar{\theta}^{4}} V_{\varphi \eta_{4} \bar{\theta}^{4}}\right\rangle=4 \int \frac{d^{2} p d^{2} q d^{2} r}{(2 \pi)^{4}} \frac{\left(p_{1}-q_{1}\right)^{2}\left(p_{0} q_{0}-p_{1} q_{1}\right) \delta^{(2)}(p+q+r)}{p^{2} q^{2}\left(r^{2}+1\right)}=0
$$

and similar cancellations happen for the other diagrams. Therefore we conclude that $W_{2, \psi_{4}}=0$ and that massless fermions are effectively decoupled at two loops.

We then move to consider massive fermions, starting from their cubic coupling to bosons

$$
\begin{aligned}
V_{z \eta \eta} & =-\epsilon^{a b c} \partial_{t} \bar{z}_{a} \eta_{b} \eta_{c}+\text { h.c. } & V_{z \eta \theta} & =-2 \epsilon^{a b c} \bar{z}_{a} \eta_{b}\left(\partial_{s}-\frac{1}{2}\right) \theta_{c}-\text { h.c. } \\
V_{\varphi \eta \theta} & =-4 i \varphi \eta_{a}\left(\partial_{s}-\frac{1}{2}\right) \bar{\theta}^{a}-h . c . & V_{x^{1} \eta \eta} & =-4 i \bar{\eta}^{a} \eta_{a}\left(\partial_{s}-\frac{1}{2}\right) x^{1} .
\end{aligned}
$$

Precisely as in the massless case, this generates five possible sunset diagrams. None of them is vanishing. We present the details of a particularly relevant example, i.e. the one involving the vertex $V_{x^{1} \eta \eta}$. This gives

$$
\begin{aligned}
-\frac{1}{2}\left\langle V_{x^{1} \eta \eta} V_{x^{1} \eta \eta}\right\rangle & =24 \int \frac{d^{2} p d^{2} q d^{2} r}{(2 \pi)^{4}} \frac{\left(p_{1}^{2}+\frac{1}{4}\right) q_{0} r_{0} \delta^{(2)}(p+q+r)}{\left(p^{2}+\frac{1}{2}\right)\left(q^{2}+\frac{1}{4}\right)\left(r^{2}+\frac{1}{4}\right)} \\
& =-\frac{3}{8} I\left(\frac{1}{2}, \frac{1}{4}, \frac{1}{4}\right)+\frac{3}{4} I\left(\frac{1}{4}\right)^{2} .
\end{aligned}
$$

We note the appearance of another integral in the class (5.8). The coefficient in front of this integral depends on the degrees of freedom of the theory and is thoroughly discussed in section (5.4). The partial results of the remaining sunset diagrams are

$$
\begin{aligned}
-\frac{1}{2}\left\langle\left(V_{z \eta \eta}+V_{z \eta \theta}\right)\left(V_{z \eta \eta}+V_{z \eta \theta}\right)\right\rangle & =3 I\left(\frac{1}{4}\right)^{2}-6 I\left(\frac{1}{4}\right) I(0) \\
-\frac{1}{2}\left\langle V_{\varphi \eta \theta} V_{\varphi \eta \theta}\right\rangle_{1 \mathrm{PI}} & =6 I\left(\frac{1}{4}\right) I(1)+\frac{3}{4} I\left(\frac{1}{4}\right)^{2} .
\end{aligned}
$$

The latter vertices can be contracted also in a non-1PI manner

$$
-\frac{1}{2}\left\langle V_{\varphi \eta \theta} V_{\varphi \eta \theta}\right\rangle_{\text {non-1PI }}=-\frac{1}{2} G_{\varphi \varphi}(0) \times 2^{6} \times 3^{2} \times \int \frac{d^{2} p}{(2 \pi)^{2}} \frac{p_{1}^{2}+\frac{1}{4}}{p^{2}+\frac{1}{4}}=-\frac{9}{2} I\left(\frac{1}{4}\right)^{2}
$$

where the factor in front of the integrals comes from the expression of the vertex and from counting the degrees of freedoms that can run in the loops. As in [42], the divergent contribution proportional to $I\left(\frac{1}{4}\right)^{2}$ cancels exactly those coming from (5.22) and (5.23).

The total cubic fermionic part reads

$$
W_{2, \text { ferm. cubic }}=-\frac{3}{8} I\left(\frac{1}{2}, \frac{1}{4}, \frac{1}{4}\right)+6 I\left(\frac{1}{4}\right) I(1)-6 I\left(\frac{1}{4}\right) I(0) .
$$


Finally we consider the fermionic double bubble diagrams. These involve the fermionic quartic vertices. However, most of the vertices appearing in the Lagrangian cannot contribute to the partition function either because the bosonic propagators are diagonal or because they would produce vanishing integrals. We present the whole list of quartic vertices in appendix B and we spell out here only the relevant ones for our computation

$$
\begin{aligned}
& V_{\varphi^{2} \eta \theta}=8 i \varphi^{2} \eta_{a}\left(\partial_{s}-\frac{1}{2}\right) \bar{\theta}^{a}-h . c . \\
& V_{z z \eta \theta}=-2 i\left[|z|^{2} \eta_{a}\left(\partial_{s}-\frac{1}{2}\right) \bar{\theta}^{a}-\bar{z}_{b} z^{a} \eta_{a}\left(\partial_{s}-\frac{1}{2}\right) \bar{\theta}^{b}\right]-\text { h.c. }
\end{aligned}
$$

Although we can build a diagram with $V_{\eta^{4}}$, fermion propagators carry one component of the loop momentum in the numerator and produce vector tadpole integrals, which vanish by parity. We conclude that the contribution from fermionic double bubble graphs is

$$
W_{2, \text { ferm. bubbles }}=-6 I\left(\frac{1}{4}\right) I(1)+6 I\left(\frac{1}{4}\right) I(0) .
$$

Summing all the partial results and reinstating the dependence on the string tension and the volume, we obtain

$$
W_{2}=\frac{V_{2}}{T}\left[\frac{1}{2} I\left(1, \frac{1}{2}, \frac{1}{2}\right)-\frac{3}{8} I\left(\frac{1}{2}, \frac{1}{4}, \frac{1}{4}\right)\right]=-\frac{1}{4} \frac{V_{2}}{T} I\left(1, \frac{1}{2}, \frac{1}{2}\right)=-\frac{K}{16 \pi^{2}} \frac{V_{2}}{T}
$$

where $T$ is defined in (1.5). Finally we can plug this expression into equation (3.9) and read out the second order of the strong coupling expansion (3.10) of the ABJM cusp anomalous dimension

$$
a_{2}=-\frac{K}{4 \pi^{2}}
$$

\subsection{The cusp anomalous dimension}

We summarize the results of our superstring computation, presenting the strong coupling expansion of the ABJM cusp anomalous dimension up to two-loop order. Reinstating the definition of the string tension (1.5) in terms of the ABJM 't Hooft coupling and plugging (4.7) and (5.29) into (3.10), we find

$$
f_{\mathrm{ABJM}}(\lambda)=\sqrt{2 \lambda}-\frac{5 \log 2}{2 \pi}-\left(\frac{K}{4 \pi^{2}}+\frac{1}{24}\right) \frac{1}{\sqrt{2 \lambda}}+\mathcal{O}\left(\lambda^{-1}\right),
$$

which is the main result of the paper. From the string dual point of view it looks convenient to define the shifted coupling

$$
\tilde{\lambda} \equiv \lambda-\frac{1}{24}
$$

in terms of which we can rewrite the scaling function more compactly as

$$
f_{\mathrm{ABJM}}(\tilde{\lambda})=\sqrt{2 \tilde{\lambda}}-\frac{5 \log 2}{2 \pi}-\frac{K}{4 \pi^{2} \sqrt{2 \tilde{\lambda}}}+\mathcal{O}\left(\tilde{\lambda}^{-1}\right)
$$




\subsection{Comparison with $A d S_{5} \times S^{5}$}

In this section we point out similarities and differences between the calculation we performed and its $A d S_{5} \times S^{5}$ analogue [42]. The starting points, i.e. the Lagrangians in AdS light-cone gauge, look rather different. Yet the final results of the two-loop computations are strikingly similar. More precisely, when written in terms of the string tension, the two expressions have exactly the same structure up to the numerical coefficients in front of the integrals. Indeed the $A d S_{5}$ computation gives

$$
W_{2}^{\left(A d S_{5}\right)}=\frac{V_{2}}{T}\left[\frac{1}{4} I\left(1, \frac{1}{2}, \frac{1}{2}\right)-\frac{1}{4} I\left(\frac{1}{2}, \frac{1}{4}, \frac{1}{4}\right)\right]
$$

which looks very similar in structure to (5.28). Furthermore, using (5.8), both combinations sum up to

$$
W_{2}=-\frac{V_{2}}{T} \frac{1}{4} I\left(1, \frac{1}{2}, \frac{1}{2}\right)
$$

and only the different relation between the string tension and the 't Hooft couplings distinguishes the final results. It is easy to trace the origin of the integrals and their coefficients back in the vertices of the Lagrangian and to understand their meaning. In particular in both computations only the sunset diagrams involving the interactions $V_{\varphi x x}$ and $V_{x \psi \psi}$ (with massive fermions) seem to effectively contribute. All other terms are also important, but just serve to cancel divergences. Hence we can now focus on the relevant interactions and point out the differences between the $A d S_{5}$ and the $A d S_{4}$ cases.

We start from the bosonic sectors. The two theories differ for the number of scalar degrees of freedom with given masses. Focussing on massive fluctuations, after gauge fixing we have one scalar with $m^{2}=1$ associated to the radial coordinate of $A d S_{d+1}$ and $(d-2)$ real scalars with $m^{2}=\frac{1}{2}$. In the metric we chose for the $A d S_{4} \times \mathbb{C P}^{3}$ background, the size of the $A d S_{4}$ part is rescaled by a factor of $r^{2}=4$. We have compensated this, parametrizing the radial coordinate as $w=e^{r \varphi}$ and introducing a factor $r$ in the fluctuation of $x^{1}$, so as to have the same normalization for their kinetic terms as in $A d S_{5} \times S^{5}$. This causes some factors $r$ to appear in interaction vertices in our Lagrangian. Apart from this, the relevant interaction vertices are exactly the same. Then, the number of $x$ fields $(d-2)$ and this factor $r$ determine the coefficient of the integral $I\left(1, \frac{1}{2}, \frac{1}{2}\right)$ appearing in equations (5.28) and (5.33).

Turning to fermions, the first striking difference between the $A d S_{5}$ and $A d S_{4}$ cases is the presence of massless ones. As pointed out at the beginning of section 5.2 their contribution is effectively vanishing at two loops (though they do contribute at first order). Focussing on massive fermions, the relevant cubic interactions giving rise to $I\left(\frac{1}{2}, \frac{1}{4}, \frac{1}{4}\right)$ look again similar in the $A d S_{4}$ and $A d S_{5}$ cases. The difference is given once more by the ratio of the radii $r$ (through the normalization of $\varphi$ and $x$ coordinates) and the number $n_{f}$ of massive fermions in the spectrum $\left(n_{f}=8\right.$ for $A d S_{5} \times S^{5}$ and $n_{f}=6$ for $\left.A d S_{4} \times \mathbb{C P}^{3}\right)$. 
The final results (5.28) and (5.33) can be re-expressed in the general form

$$
\begin{aligned}
W_{2}^{\left(A d S_{d+1}\right)} & =\frac{V_{2}}{T} \frac{(d-2) r^{2}}{8}\left[I\left(1, \frac{1}{2}, \frac{1}{2}\right)-\frac{n_{f}}{8} I\left(\frac{1}{2}, \frac{1}{4}, \frac{1}{4}\right)\right] \\
& =\frac{V_{2}}{T} \frac{(d-2) r^{2}}{8}\left(1-\frac{n_{f}}{4}\right) I\left(1, \frac{1}{2}, \frac{1}{2}\right), \quad d=3,4,
\end{aligned}
$$

where the cases at hand are $d=4, n_{f}=8, r=1$ for $\mathcal{N}=4$ SYM and $d=3, n_{f}=6, r=2$ for ABJM.

\section{Concluding remarks}

In this work we have computed the cusp anomalous dimension of ABJM theory up to second order in its strong coupling expansion. This result has been determined considering the $A d S_{4} \times \mathbb{C P}^{3} \kappa$-symmetry gauge-fixed action of $[45,46]$ and studying its fluctuations about the null cusp background (3.1), which is a classical solution thereof. As in the $A d S_{5} \times S^{5}$ counterpart of this calculation [42], the AdS light-cone gauge approach [49] makes the explicit evaluation rather manageable, allowing us to push the expansion of the string partition function up to second order.

While at one loop we confirm a known result $[28,32,36]$, at two-loops we provide a new important piece of data, see (1.6), which we combine with a proposal based on the Bethe Ansatz of $A d S_{4} / \mathrm{CFT}_{3}$ [4] to give our two-loop correction to the so-called interpolating function $h(\lambda)$ of ABJM theory, equation (1.10). Importantly, the recent conjecture of [66] for an all-order expression of $h(\lambda)$, implicitly given in terms of a non-trivial hypergeometric function, agrees with our result, which is a relevant perturbative test of validity for the conjecture. In particular, at this level of perturbation theory we must implement in our calculation a "correction" to the string tension in terms of the 't Hooft coupling, which was pointed out in [64] to be due to higher order corrections (in curvature) to the background. We show that the strong coupling two-loop correction for $h(\lambda)$ is only due to the anomalous shift of the curvature radius in the Type IIA description [64]. This supports the observation in [66] on the origin of the shift appearing in its proposal (1.13), which knows nothing about the gravity side but coincides in fact with the correction of [64].

In perspective, we observe that the light-cone gauge approach could be pushed to a much stronger check of (1.11), by testing its finite coupling regime. Following [79], one could discretize the light-cone Lagrangian (3.4), put it on a lattice and perform numerical simulations to determine the ABJM scaling function in terms of the coupling constant, for any value thereof. By comparison with the same results for $\mathcal{N}=4 \mathrm{SYM}$ one could then provide numerical values of $h(\lambda)$ at some finite values of $\lambda$, which could then be contrasted with (1.11).

The manifest cancellation of UV divergences that we find here provides a direct demonstration of the quantum consistency of the $A d S_{4} \times \mathbb{C P}^{3}$ action of $[45,46]$, and shows that it can be readily used for non-trivial strong coupling computations in the $A d S_{4} / \mathrm{CFT}_{3}$ framework (following for example $[52,53]$ ). In particular, the consistency of the result with predictions coming from integrability, the conjecture [66] and the "corrected" dictionary of [64] can be taken as evidence, albeit indirect, of quantum integrability for the Type IIA $A d S_{4} \times \mathbb{C P}^{3}$ superstring in this gauge. 


\section{Acknowledgments}

It is a pleasure to thank Alessandra Cagnazzo, Andrea Cavaglià, Ben Hoare, Valentina G.M.Puletti, Nikolay Gromov, Radu Roiban, Roberto Tateo and Arkady Tseytlin for discussions, and in particular Ben Hoare for useful comments on the draft. The work of $\mathrm{LB}, \mathrm{AB}, \mathrm{VF}$ and EV is funded by DFG via the Emmy Noether Program "Gauge Fields from Strings".

\section{A Lagrangian in the Wess-Zumino type parametrization}

In this appendix we rewrite the Lagrangian (2.5) in a form that resembles the Wess-Zumino type parametrization introduced in [49], and compare it to the $A d S_{5} \times S^{5}$ case. In [49] the authors found two possible ways to eliminate the fermion rotation (2.12), either by a change of parametrization for $S^{5}$ or by the introduction of a covariant derivative for the terms quadratic in fermions. Here we explore only the second option and we leave the first one for future development. We first introduce a collective index for upper and lower indices so that

$$
\eta_{\hat{a}}=\left(\begin{array}{c}
\eta_{a} \\
\bar{\eta}^{a}
\end{array}\right) .
$$

In this notation the action of the matrix $T$ on the fermions (2.12) can be rewritten as

$$
\hat{\eta}_{\hat{a}}=T_{\hat{a}}^{\hat{b}} \eta_{\hat{b}}
$$

where the matrix $T_{\hat{a}}^{\hat{b}}$ is given in (2.17). We also introduce the shorthand notation

$$
\partial_{i} \eta_{a} \bar{\eta}^{a}-\eta_{a} \partial_{i} \bar{\eta}^{a}=-\eta^{\hat{a}} \partial_{i} \eta_{\hat{a}}
$$

where $\eta^{\hat{a}}=\left(\bar{\eta}^{a}, \eta_{a}\right)$. In [49] a recipe for going from the Killing parametrization to a WessZumino type gauge was given, which consists of rotating back the fermions. This generates additional terms coming from derivatives that can be reabsorbed into a covariant derivative. In particular, we apply the transformation

$$
\eta_{\hat{a}} \rightarrow\left(T^{-1}\right)_{\hat{a}}^{\hat{b}} \eta_{\hat{b}}
$$

In contrast with the $A d S_{5} \times S^{5}$ case the matrix $T$ is not block diagonal, therefore one has $\eta^{\hat{a}} \partial_{i} \eta_{\hat{a}}=\hat{\eta}^{\hat{a}} \hat{\partial}_{i} \eta_{\hat{a}}$, where it is crucial to use hatted indices. This transformation removes all the hats from fermions, at the price of introducing the covariant derivative

$$
D=d-\Omega,
$$

where $\Omega \equiv \Omega_{\hat{a}}{ }^{\hat{b}}=d T_{\hat{a}}{ }^{\hat{c}}\left(T^{-1}\right)_{\hat{c}}^{\hat{b}}$ and $d \Omega-\Omega \wedge \Omega=0$. More explicitly, ${ }^{16}$ the (matrix) Cartan form entering the definition of the (dimensionally reduced) supercoset element reads

$$
\Omega_{\hat{a}}^{\hat{b}}=i\left(\begin{array}{cc}
\Omega_{a}^{b}-\delta_{a}^{b} \Omega_{c}^{c} & \epsilon_{a c b} \Omega^{c} \\
-\epsilon^{a c b} \Omega_{c} & -\Omega_{b}^{a}+\delta_{b}^{a} \Omega_{c}^{c}
\end{array}\right),
$$

\footnotetext{
${ }^{16}$ The matrix $\Omega$ was already introduced in [76] however there it was defined as $\Omega_{\hat{a}}{ }^{\hat{b}}=i T_{\hat{a}}{ }^{\hat{c}} d T^{-1}{ }_{\hat{c}}^{\hat{b}}=$ $-i d T_{\hat{a}}{ }^{\hat{c}} T^{-1}{ }_{\hat{c}}^{\hat{b}}$, differing from ours by a factor of $i$. To make contact with the expressions of [76] we add such a factor in formula (A.6).
} 
with components given by

$$
\begin{aligned}
& \Omega_{a}{ }^{b}=i \frac{(1-\cos |z|)}{|z|^{2}}\left(\bar{z}_{a} d z^{b}-d \bar{z}_{a} z^{b}\right)-i \bar{z}_{a} z^{b} \frac{(1-\cos |z|)^{2}}{2|z|^{4}}\left(d z^{c} \bar{z}_{c}-z^{c} d \bar{z}_{c}\right), \\
& \Omega_{a}=d \bar{z}_{a} \frac{\sin |z|}{|z|}+\bar{z}_{a} \frac{\sin |z|(1-\cos |z|)}{2|z|^{3}}\left(d z^{c} \bar{z}_{c}-z^{c} d \bar{z}_{c}\right)+\bar{z}_{a}\left(\frac{1}{|z|}-\frac{\sin |z|}{|z|^{2}}\right) d|z|, \\
& \Omega^{a}=d z^{a} \frac{\sin |z|}{|z|}+z^{a} \frac{\sin |z|(1-\cos |z|)}{2|z|^{3}}\left(z^{c} d \bar{z}_{c}-d z^{c} \bar{z}_{c}\right)+z^{a}\left(\frac{1}{|z|}-\frac{\sin |z|}{|z|^{2}}\right) d|z| .
\end{aligned}
$$

Above, $\Omega_{c}{ }^{c}$ is the trace of (A.7) and is related to $\tilde{\Omega}_{c}^{c}$ defined in (2.16) via $\tilde{\Omega}_{c}^{c}=2 \Omega_{c}{ }^{c}$.

We can also decompose the matrix $\Omega$ in order to separate the contributions from the vielbein and from the spin connection ${ }^{17}$

$$
\Omega_{\hat{a}}^{\hat{b}}=\Omega^{\hat{c}}\left(E_{\hat{c}}\right)_{\hat{a}}^{\hat{b}}+\Omega_{d}^{c}\left(J_{c}^{d}\right)_{\hat{a}}^{\hat{b}}
$$

with $^{18}$

$$
\left(E_{\hat{c}}\right)_{\hat{a}}^{\hat{b}}=i\left(\begin{array}{cc}
0 & \epsilon_{a c b} \\
-\epsilon^{a c b} & 0
\end{array}\right) \quad\left(J_{c}^{d}\right)_{\hat{a}}^{\hat{b}}=i\left(\begin{array}{cc}
\delta_{a}^{d} \delta_{c}^{b}-\delta_{a}^{b} \delta_{c}^{d} & 0 \\
0 & -\delta_{b}^{d} \delta_{c}^{a}+\delta_{b}^{a} \delta_{c}^{d}
\end{array}\right) .
$$

This decomposition provides a way to project out the spin connection and find the exact relation between the vielbein $\Omega_{\hat{a}}$ and the matrix $\Omega$

$$
\Omega_{\hat{c}}=\frac{1}{2} \operatorname{Tr}\left(E_{\hat{c}} \Omega\right)
$$

After having introduced all the necessary ingredients, we are ready to rewrite the Lagrangian in a form which resembles the $A d S_{5} \times S^{5}$ case. We separate it into

$$
L=L_{B}+L_{F}^{(2)}+L_{F}^{(4)}
$$

where the bosonic contribution is simply given by the standard bosonic sigma model with $A d S_{4} \times \mathbb{C P}^{3}$ as target space

$$
L_{B}=\gamma^{i j}\left[\frac{e^{-4 \varphi}}{4}\left(\partial_{i} x^{+} \partial_{j} x^{-}+\partial_{i} x^{1} \partial_{j} x^{1}\right)+\partial_{i} \varphi \partial_{j} \varphi+\Omega^{a}{ }_{i} \Omega_{a j}\right]
$$

where the vielbein $\Omega^{a}{ }_{i}$ are defined in the natural way $\Omega^{a}=\Omega^{a}{ }_{i} d \sigma^{i}$ with $\sigma^{i}=(\tau, \sigma)$. Notice also that $\Omega^{\hat{a}_{i}} \Omega_{\hat{a} j}=2 \Omega^{a}{ }_{i} \Omega_{a j}$ for the symmetry of the worldsheet metric. The quadratic

\footnotetext{
${ }^{17} \mathrm{~A}$ similar procedure was applied in [49] where in that case the decomposition is expressed in terms of the $\mathrm{SO}(5) \gamma$-matrices.

${ }^{18}$ Let us stress that the meaning of the first term of equation (A.10) in matrix form is the following

$$
\Omega^{\hat{c}}\left(E_{\hat{c}}\right)_{\hat{a}}^{\hat{b}}=\left(\begin{array}{cc}
\Omega^{c}\left(E_{c}\right)_{a}{ }^{b}+\Omega_{c}\left(E^{c}\right)_{a}{ }^{b} & \Omega^{c}\left(E_{c}\right)_{a b}+\Omega_{c}\left(E^{c}\right)_{a b} \\
\Omega^{c}\left(E_{c}\right)^{a b}+\Omega_{c}\left(E^{c}\right)^{a b} & \Omega^{c}\left(E_{c}\right)^{a}{ }_{b}+\Omega_{c}\left(E^{c}\right)^{a}{ }_{b}
\end{array}\right)
$$

and the explicit expression of $\left(E_{\hat{c}}\right)_{\hat{a}}^{\hat{b}}$ shows that the only non-vanishing elements are $\left(E_{c}\right)_{a b}$ and $\left(E^{c}\right)^{a b}$.
} 
part in the fermion fields can be expressed as

$$
\begin{aligned}
L_{F}^{(2)}= & -2 e^{-4 \varphi} \partial_{i} x^{+}\left[\frac{i}{2} \gamma^{i j}\left(\eta^{\hat{a}} D_{j} \eta_{\hat{a}}+\theta^{\hat{a}} D_{j} \theta_{\hat{a}}-2 \Omega_{j}^{\hat{c}} \eta E_{\hat{c}} \eta\right)+\varepsilon^{i j} \eta^{\hat{a}} C_{\hat{a}}^{\hat{b}}\left(D_{j} \theta_{\hat{b}}+e^{-2 \varphi} \eta_{\hat{b}} \partial_{j} x^{1}\right)\right. \\
& +\frac{i}{2} \gamma^{i j}\left(\bar{\eta}^{4} \partial_{j} \eta_{4}+\bar{\theta}^{4} \partial_{j} \theta_{4}-4 i \eta_{a} \Omega_{j}^{a} \eta_{4}+2 i \Omega_{a}^{a}{ }_{j} \Theta-h . c .\right) \\
& \left.+\frac{1}{2} \varepsilon^{i j}\left(\bar{\eta}^{4} \partial_{j} \theta_{4}-\bar{\theta}^{4} \partial_{j} \eta_{4}+4 i \eta_{a} \Omega_{j}^{a} \theta_{4}+2 i \Omega_{a}^{a}{ }_{j} \tilde{\Theta}-e^{-2 \varphi} \Theta \partial_{j} x^{1}+h . c .\right)\right] . \quad \text { (A.16) }
\end{aligned}
$$

Here we have introduced the charge conjugation matrix $C$, given explicitly by ${ }^{19}$

$$
C_{\hat{a}}^{\hat{b}}=\left(\begin{array}{cc}
\delta_{a}^{b} & 0 \\
0 & -\delta_{b}^{a}
\end{array}\right),
$$

and the combinations $\Theta=\theta_{4} \bar{\theta}^{4}+\eta_{4} \bar{\eta}^{4}$ and $\tilde{\Theta}=\theta_{4} \bar{\eta}^{4}-\eta_{4} \bar{\theta}^{4}$. The first line of this Lagrangian (A.16) closely resembles expression (1.6) of [49], that is the $A d S_{5} \times S^{5}$ Lagrangian in Wess-Zumino type parametrization. This is the part of the Lagrangian that does not contain the fermions $\eta_{4}$ and $\theta_{4}$, which emerge [45] when obtaining the $A d S_{4} \times \mathbb{C P}^{3}$ action from dimensional reduction of the $A d S_{4} \times S^{7}$ supermembrane action. The main difference with respect to $A d S_{5} \times S^{5}$ is that the $\mathrm{SU}(4)$ R-symmetry is not explicitly realized on the fermionic Lagrangian (A.16). This feature is inherited by the quantum fluctuations around the light-like cusp. As a result of the broken symmetry, the spectrum contains fermionic degrees of freedom with different masses (one gets 6 massive and 2 massless excitations). Our one- and two-loop calculations have explicitly shown that the role of the massless fermions $\left(\tilde{\eta}_{4}\right.$ and $\left.\tilde{\theta}_{4}\right)$ is crucial for compensating the bosonic degrees of freedom, making the one-loop partition function UV-finite. At two loops their interactions with the other excitations would in principle start playing a part. Nevertheless it turns out that the massless fermions decouple from the computation and do not contribute to the two-loop result.

The last term of the superstring Lagrangian is quartic in fermions

$$
L_{F}^{(4)}=4 e^{-8 \varphi} \gamma^{i j} \partial_{i} x^{+} \partial_{j} x^{+}\left[\left(\eta_{a} \bar{\eta}^{a}\right)^{2}+2 \varepsilon^{a b c} \eta_{a} \eta_{b} \eta_{c} \eta_{4}+2 \eta_{4} \bar{\eta}^{4} \eta_{a} \bar{\eta}^{a}-\Theta^{2}+h . c .\right] .
$$

As discussed for the quadratic part, the first terms clearly reminds the expression for $A d S_{5} \times S^{5}$ (equation (1.10) of [49]), whereas the others contain the non-trivial interactions of $\eta_{4}$ and $\theta_{4}$.

\section{B Details on the expanded Lagrangian}

In this appendix we provide the details of the Lagrangian (3.4) expanded up to quartic order. As in section 5 we list the vertices as they appear in $\mathcal{L}_{\text {int }}$, namely with an extra factor $\frac{1}{2}$ with respect to the original Lagrangian. We drop tildas, understanding that we are dealing with the fluctuation fields of (3.4). The cubic vertices are

$$
V_{\varphi x^{1} x^{1}}=-4 \varphi\left[\left(\partial_{s}-\frac{1}{2}\right) x^{1}\right]^{2} \quad V_{\varphi^{3}}=2 \varphi\left[\left(\partial_{t} \varphi\right)^{2}-\left(\partial_{s} \varphi\right)^{2}\right] \quad V_{\varphi|z|^{2}}=2 \varphi\left[\left|\partial_{t} z\right|^{2}-\left|\partial_{s} z\right|^{2}\right]
$$

\footnotetext{
${ }^{19}$ The fact that the matrix is diagonal and not anti-diagonal is a consequence of our conventions for grouping the spinors. Notice also that for our conventions $\eta^{\hat{a}} \eta_{\hat{a}}=0$ whereas $\eta^{\hat{a}} C_{\hat{a}}{ }^{\hat{b}} \eta_{\hat{b}}=-2 \eta_{a} \bar{\eta}^{a}$.
} 


$$
\begin{aligned}
V_{z \eta \eta} & =-\epsilon^{a b c} \partial_{t} \bar{z}_{a} \eta_{b} \eta_{c}+\text { h.c. } & V_{z \eta \theta} & =-2 \epsilon^{a b c} \bar{z}_{a} \eta_{b}\left(\partial_{s}-\frac{1}{2}\right) \theta_{c}-\text { h.c. } \\
V_{\varphi \eta \theta} & =-4 i \varphi \eta_{a}\left(\partial_{s}-\frac{1}{2}\right) \bar{\theta}^{a}-h . c . & V_{x^{1} \eta \eta} & =-4 i \bar{\eta}^{a} \eta_{a}\left(\partial_{s}-\frac{1}{2}\right) x^{1} \\
V_{z \eta_{a} \eta_{4}} & =-2 \partial_{t} z^{a} \eta_{a} \eta_{4}+h . c . & V_{z \eta_{a} \theta_{4}} & =2 \partial_{s} z^{a} \eta_{a} \theta_{4}-h . c . \\
V_{\varphi \eta_{4} \bar{\theta}^{4}} & =-2 i \varphi\left(\bar{\theta}^{4} \partial_{s} \eta_{4}-\partial_{s} \bar{\theta}^{4} \eta_{4}\right)-h . c . & V_{x^{1} \bar{\psi}^{4} \psi_{4}} & =-2 i\left(\bar{\eta}^{4} \eta_{4}+\bar{\theta}^{4} \theta_{4}\right)\left(\partial_{s}-\frac{1}{2}\right) x^{1}
\end{aligned}
$$

The quartic vertices read

$$
\begin{aligned}
& V_{z^{4}}=\frac{1}{6}\left[\left(\bar{z}_{a} \partial_{t} z^{a}\right)^{2}+\left(\bar{z}_{a} \partial_{s} z^{a}\right)^{2}+\left(z^{a} \partial_{t} \bar{z}_{a}\right)^{2}+\left(z^{a} \partial_{s} \bar{z}_{a}\right)^{2}\right. \\
& \left.-|z|^{2}\left(\left|\partial_{t} z\right|^{2}+\left|\partial_{s} z\right|^{2}\right)-\left|\bar{z}_{a} \partial_{t} z^{a}\right|^{2}-\left|\bar{z}_{a} \partial_{s} z^{a}\right|^{2}\right] \\
& V_{\varphi^{2} x^{1} x^{1}}=16 \varphi^{2}\left[\left(\partial_{s}-\frac{1}{2}\right) x^{1}\right]^{2} \quad V_{\varphi^{4}}=4 \varphi^{2}\left[\left(\partial_{t} \varphi\right)^{2}+\left(\partial_{s} \varphi\right)^{2}+\frac{1}{6} \varphi^{2}\right] \\
& V_{\varphi^{2}|z|^{2}}=4 \varphi^{2}\left[\left|\partial_{t} z\right|^{2}+\left|\partial_{s} z\right|^{2}\right] \quad V_{\dot{z} \bar{z} \bar{\psi}^{4} \psi_{4}}=-2 i\left(\bar{\eta}^{4} \eta_{4}+\bar{\theta}^{4} \theta_{4}\right) \bar{z}_{b} \partial_{t} z^{b}+\text { h.c. } \\
& V_{\eta^{2} \eta_{4} \bar{\eta}_{4}}=8 \bar{\eta}^{4} \eta_{4} \bar{\eta}^{a} \eta_{a} \quad V_{z^{\prime} \bar{z} \bar{\psi}^{4} \psi_{4}}=-2 i\left(\bar{\eta}^{4} \theta_{4}-\bar{\theta}^{4} \eta_{4}\right) \bar{z}_{b} \partial_{s} z^{b}-\text { h.c. } \\
& V_{\eta^{4}}=4\left(\bar{\eta}^{a} \eta_{a}\right)^{2} \\
& V_{\eta_{4} \bar{\eta}_{4} \theta_{4} \bar{\theta}_{4}}=-8 \bar{\eta}^{4} \eta_{4} \bar{\theta}^{4} \theta_{4} \\
& V_{\varphi^{2} \eta_{4} \bar{\theta}^{4}}=4 i \varphi^{2}\left(\bar{\theta}^{4} \partial_{s} \eta_{4}-\partial_{s} \bar{\theta}^{4} \eta_{4}\right)-\text { h.c. } \\
& V_{\eta^{3} \eta_{4}}=4 \epsilon^{a b c} \eta_{a} \eta_{b} \eta_{c} \eta_{4}+\text { h.c. } \\
& V_{\varphi x^{1} \bar{\psi}^{4} \psi_{4}}=12 i \varphi\left(\bar{\eta}^{4} \eta_{4}+\bar{\theta}^{4} \theta_{4}\right)\left(\partial_{s}-\frac{1}{2}\right) x^{1} \\
& V_{z z \bar{\eta}^{a} \eta_{4}}=-2 i \epsilon_{a b c} \partial_{t} z^{a} z^{b} \bar{\eta}^{c} \eta_{4}+\text { h.c. } \\
& V_{\varphi z \eta_{a} \theta_{4}}=-8 \varphi \partial_{s} z^{a} \eta_{a} \theta_{4}-h . c . \quad \quad V_{\varphi z \eta \theta}=8 \varphi \epsilon^{a b c} \bar{z}_{a} \eta_{b}\left(\partial_{s}-\frac{1}{2}\right) \theta_{c}-\text { h.c. } \\
& V_{z z \bar{\eta}^{a} \theta_{4}}=2 i \epsilon_{a b c} \partial_{s} z^{a} z^{b} \bar{\eta}^{c} \theta_{4}-h . c . \quad V_{z z \eta \eta}=-2 i\left(\bar{z}_{a} \partial_{t} z^{a} \bar{\eta}^{b} \eta_{b}-\bar{z}_{b} \partial_{t} z^{a} \bar{\eta}^{b} \eta_{a}\right)+\text { h.c. } \\
& V_{\varphi x^{1} \eta \eta}=24 i \varphi \bar{\eta}^{a} \eta_{a}\left(\partial_{s}-\frac{1}{2}\right) x^{1} \\
& V_{z z \eta \theta}=-2 i\left[|z|^{2} \eta_{a}\left(\partial_{s}-\frac{1}{2}\right) \bar{\theta}^{a}-\bar{z}_{b} z^{a} \eta_{a}\left(\partial_{s}-\frac{1}{2}\right) \bar{\theta}^{b}\right]-\text { h.c. } \\
& V_{\varphi^{2} \eta \theta}=8 i \varphi^{2} \eta_{a}\left(\partial_{s}-\frac{1}{2}\right) \bar{\theta}^{a}-\text { h.c. } \quad V_{x^{1} z \eta \eta}=-4\left(\partial_{s}-\frac{1}{2}\right) x^{1} \epsilon^{a b c} \bar{z}_{a} \eta_{b} \eta_{c}-\text { h.c. }
\end{aligned}
$$

\section{Integral reductions}

In this appendix we provide the relevant tensor integral reductions in two dimensions that we used in the computation of the two-loop correction to the partition function. We define the two basic scalar integrals

$$
\begin{aligned}
I\left(m^{2}\right) & \equiv \int \frac{d^{2} p}{(2 \pi)^{2}} \frac{1}{p^{2}+m^{2}} \\
I\left(m_{1}^{2}, m_{2}^{2}, m_{3}^{2}\right) & \equiv \int \frac{d^{2} p d^{2} q d^{2} r}{(2 \pi)^{4}} \frac{\delta^{(2)}(p+q+r)}{\left(p^{2}+m_{1}^{2}\right)\left(q^{2}+m_{2}^{2}\right)\left(r^{2}+m_{3}^{2}\right)} .
\end{aligned}
$$

Then we have (the factors $(2 \pi)^{4}$ in the denominator of the integrands are understood)

$$
\begin{aligned}
& \int \frac{d^{2} p d^{2} q d^{2} r p^{\mu} q^{\nu} \delta^{(2)}(p+q+r)}{\left(p^{2}+m_{1}^{2}\right)\left(q^{2}+m_{2}^{2}\right)\left(r^{2}+m_{3}^{2}\right)}= \\
& =\frac{\delta^{\mu \nu}}{4}\left[I\left(m_{1}^{2}\right) I\left(m_{2}^{2}\right)-I\left(m_{1}^{2}\right) I\left(m_{3}^{2}\right)-I\left(m_{2}^{2}\right) I\left(m_{3}^{2}\right)+\left(m_{1}^{2}+m_{2}^{2}-m_{3}^{2}\right) I\left(m_{1}^{2}, m_{2}^{2} ; m_{3}^{2}\right)\right] \\
& I_{\mu}^{\mu}\left(m_{1}^{2}, m_{2}^{2} ; m_{3}^{2}\right)=\int \frac{d^{2} p d^{2} q d^{2} r(p \cdot q) \delta^{(2)}(p+q+r)}{\left(p^{2}+m_{1}^{2}\right)\left(q^{2}+m_{2}^{2}\right)\left(r^{2}+m_{3}^{2}\right)}= \\
& =\frac{1}{2}\left[I\left(m_{1}^{2}\right) I\left(m_{2}^{2}\right)-I\left(m_{1}^{2}\right) I\left(m_{3}^{2}\right)-I\left(m_{2}^{2}\right) I\left(m_{3}^{2}\right)+\left(m_{1}^{2}+m_{2}^{2}-m_{3}^{2}\right) I\left(m_{1}^{2}, m_{2}^{2} ; m_{3}^{2}\right)\right]
\end{aligned}
$$




$$
\begin{aligned}
& \int \frac{d^{2} p d^{2} q d^{2} r p^{\mu} p^{\nu} \delta^{(2)}(p+q+r)}{\left(p^{2}+m_{1}^{2}\right)\left(q^{2}+m_{2}^{2}\right)\left(r^{2}+m_{3}^{2}\right)}=\frac{\delta^{\mu \nu}}{2}\left[I\left(m_{2}^{2}\right) I\left(m_{3}^{2}\right)-m_{1}^{2} I\left(m_{1}^{2}, m_{2}^{2} ; m_{3}^{2}\right)\right] \\
& J \equiv \int \frac{d^{2} p d^{2} q d^{2} r p^{2} q^{2} \delta^{(2)}(p+q+r)}{\left(p^{2}+m_{1}^{2}\right)\left(q^{2}+m_{2}^{2}\right)\left(r^{2}+m_{3}^{2}\right)}=m_{1}^{2} m_{2}^{2} I\left(m_{1}^{2}, m_{2}^{2} ; m_{3}^{2}\right)-m_{1}^{2} I\left(m_{1}^{2}\right) I\left(m_{3}^{2}\right) \\
& -m_{2}^{2} I\left(m_{2}^{2}\right) I\left(m_{3}^{2}\right) \\
& K \equiv \int \frac{d^{2} p d^{2} q d^{2} r(p \cdot q)^{2} \delta^{(2)}(p+q+r)}{\left(p^{2}+m_{1}^{2}\right)\left(q^{2}+m_{2}^{2}\right)\left(r^{2}+m_{3}^{2}\right)}=\frac{1}{2}\left[-m_{2}^{2} I\left(m_{2}^{2}\right) I\left(m_{3}^{2}\right)-m_{1}^{2} I\left(m_{1}^{2}\right) I\left(m_{3}^{2}\right)+\right. \\
& \left.+\left(m_{1}^{2}+m_{2}^{2}-m_{3}^{2}\right) I_{\mu}^{\mu}\left(m_{1}^{2}, m_{2}^{2} ; m_{3}^{2}\right)\right] \\
& \int \frac{d^{2} p d^{2} q d^{2} r p^{\mu} p^{\nu} q^{\rho} q^{\sigma} \delta^{(2)}(p+q+r)}{\left(p^{2}+m_{1}^{2}\right)\left(q^{2}+m_{2}^{2}\right)\left(r^{2}+m_{3}^{2}\right)}=\left(\frac{3}{8} J-\frac{1}{4} K\right) \delta^{\mu \nu} \delta^{\rho \sigma} \\
& +\left(\frac{1}{4} K-\frac{1}{8} J\right)\left(\delta^{\mu \rho} \delta^{\nu \sigma}+\delta^{\mu \sigma} \delta^{\nu \rho}\right) \\
& \int \frac{d^{2} p d^{2} q d^{2} r p^{\mu} p^{\nu} p^{\rho} q^{\sigma} \delta^{(2)}(p+q+r)}{\left(p^{2}+m_{1}^{2}\right)\left(q^{2}+m_{2}^{2}\right)\left(r^{2}+m_{3}^{2}\right)}=\frac{1}{8}\left(\delta^{\mu \nu} \delta^{\rho \sigma}+\delta^{\mu \rho} \delta^{\nu \sigma}+\delta^{\mu \sigma} \delta^{\nu \rho}\right) \\
& {\left[m_{2}^{2} I\left(m_{2}^{2}\right) I\left(m_{3}^{2}\right)-m_{1}^{2} I_{\mu}^{\mu}\left(m_{1}^{2}, m_{2}^{2} ; m_{3}^{2}\right)\right]} \\
& L \equiv \int \frac{d^{2} p d^{2} q d^{2} r p^{2}(q \cdot r) \delta^{(2)}(p+q+r)}{\left(p^{2}+m_{1}^{2}\right)\left(q^{2}+m_{2}^{2}\right)\left(r^{2}+m_{3}^{2}\right)}=-m_{1}^{2} I_{\mu}^{\mu}\left(m_{3}^{2}, m_{2}^{2} ; m_{1}^{2}\right) \\
& M \equiv \int \frac{d^{2} p d^{2} q d^{2} r(p \cdot q)(p \cdot r) \delta^{(2)}(p+q+r)}{\left(p^{2}+m_{1}^{2}\right)\left(q^{2}+m_{2}^{2}\right)\left(r^{2}+m_{3}^{2}\right)}=\frac{1}{2}\left[\left(m_{1}^{2}+m_{3}^{2}-m_{2}^{2}\right) I_{\mu}^{\mu}\left(m_{1}^{2}, m_{2}^{2} ; m_{3}^{2}\right)+\right. \\
& \left.+m_{1}^{2} I\left(m_{1}^{2}\right) I\left(m_{3}^{2}\right)-m_{2}^{2} I\left(m_{2}^{2}\right) I\left(m_{3}^{2}\right)\right] \\
& \int \frac{d^{2} p d^{2} q d^{2} r p^{\mu} p^{\nu} q^{\rho} r^{\sigma} \delta^{(2)}(p+q+r)}{\left(p^{2}+m_{1}^{2}\right)\left(q^{2}+m_{2}^{2}\right)\left(r^{2}+m_{3}^{2}\right)}=\left(\frac{3}{8} L-\frac{1}{4} M\right) \delta^{\mu \nu} \delta^{\rho \sigma} \\
& +\left(\frac{1}{4} M-\frac{1}{8} L\right)\left(\delta^{\mu \rho} \delta^{\nu \sigma}+\delta^{\mu \sigma} \delta^{\nu \rho}\right) .
\end{aligned}
$$

Open Access. This article is distributed under the terms of the Creative Commons Attribution License (CC-BY 4.0), which permits any use, distribution and reproduction in any medium, provided the original author(s) and source are credited.

\section{References}

[1] O. Aharony, O. Bergman, D.L. Jafferis and J. Maldacena, $N=6$ superconformal Chern-Simons-matter theories, M2-branes and their gravity duals, JHEP 10 (2008) 091 [arXiv:0806.1218] [INSPIRE].

[2] J.M. Maldacena, The large- $N$ limit of superconformal field theories and supergravity, Int. J. Theor. Phys. 38 (1999) 1113 [hep-th/9711200] [INSPIRE].

[3] J.A. Minahan and K. Zarembo, The Bethe ansatz for superconformal Chern-Simons, JHEP 09 (2008) 040 [arXiv: 0806.3951] [INSPIRE].

[4] N. Gromov and P. Vieira, The all loop AdS4/CFT3 Bethe ansatz, JHEP 01 (2009) 016 [arXiv:0807.0777] [INSPIRE]. 
[5] T. Klose, Review of AdS/CFT Integrability, Chapter IV.3: $N=6$ Chern-Simons and Strings on $A d S_{4} \times C P^{3}$, Lett. Math. Phys. 99 (2012) 401 [arXiv:1012.3999] [INSPIRE].

[6] A. Cavaglià, D. Fioravanti, N. Gromov and R. Tateo, The Quantum Spectral Curve of the ABJM theory, Phys. Rev. Lett. 113 (2014) 021601 [arXiv:1403.1859] [InSPIRE].

[7] J.M. Maldacena, Wilson loops in large-N field theories, Phys. Rev. Lett. 80 (1998) 4859 [hep-th/9803002] [INSPIRE].

[8] S.S. Gubser, I.R. Klebanov and A.M. Polyakov, A semi-classical limit of the gauge/string correspondence, Nucl. Phys. B 636 (2002) 99 [hep-th/0204051] [INSPIRE].

[9] M. Kruczenski, A note on twist two operators in $N=4 S Y M$ and Wilson loops in Minkowski signature, JHEP 12 (2002) 024 [hep-th/0210115] [INSPIRE].

[10] D. Correa, J. Henn, J. Maldacena and A. Sever, An exact formula for the radiation of a moving quark in $N=4$ super Yang-Mills, JHEP 06 (2012) 048 [arXiv:1202.4455] [INSPIRE].

[11] D. Correa, J. Maldacena and A. Sever, The quark anti-quark potential and the cusp anomalous dimension from a TBA equation, JHEP 08 (2012) 134 [arXiv:1203.1913] [INSPIRE].

[12] N. Gromov and A. Sever, Analytic Solution of Bremsstrahlung TBA, JHEP 11 (2012) 075 [arXiv: 1207.5489] [INSPIRE].

[13] D.M. Hofman and J.M. Maldacena, Giant Magnons, J. Phys. A 39 (2006) 13095 [hep-th/0604135] [INSPIRE].

[14] T. Klose, T. McLoughlin, J.A. Minahan and K. Zarembo, World-sheet scattering in $A d S_{5} \times S^{5}$ at two loops, JHEP 08 (2007) 051 [arXiv: 0704.3891] [INSPIRE].

[15] D. Berenstein and D. Trancanelli, S-duality and the giant magnon dispersion relation, Eur. Phys. J. C 74 (2014) 2925 [arXiv:0904.0444] [INSPIRE].

[16] D. Gaiotto, S. Giombi and X. Yin, Spin Chains in $N=6$ Superconformal Chern-Simons-Matter Theory, JHEP 04 (2009) 066 [arXiv:0806.4589] [INSPIRE].

[17] G. Grignani, T. Harmark and M. Orselli, The $\mathrm{SU}(2) \times \mathrm{SU}(2)$ sector in the string dual of $N=6$ superconformal Chern-Simons theory, Nucl. Phys. B 810 (2009) 115 [arXiv: 0806.4959] [INSPIRE].

[18] T. Nishioka and T. Takayanagi, On Type IIA Penrose Limit and $\mathcal{N}=6$ Chern-Simons Theories, JHEP 08 (2008) 001 [arXiv:0806.3391] [INSPIRE].

[19] J.A. Minahan, O. Ohlsson Sax and C. Sieg, Magnon dispersion to four loops in the ABJM and ABJ models, J. Phys. A 43 (2010) 275402 [arXiv:0908.2463] [InSPIRE].

[20] J.A. Minahan, O. Ohlsson Sax and C. Sieg, Anomalous dimensions at four loops in $N=6$ superconformal Chern-Simons theories, Nucl. Phys. B 846 (2011) 542 [arXiv:0912.3460] [INSPIRE].

[21] M. Leoni et al., Superspace calculation of the four-loop spectrum in $N=6$ supersymmetric Chern-Simons theories, JHEP 12 (2010) 074 [arXiv:1010.1756] [INSPIRE].

[22] A.V. Belitsky, A.S. Gorsky and G.P. Korchemsky, Logarithmic scaling in gauge/string correspondence, Nucl. Phys. B 748 (2006) 24 [hep-th/0601112] [INSPIRE]. 
[23] M. Kruczenski, R. Roiban, A. Tirziu and A.A. Tseytlin, Strong-coupling expansion of cusp anomaly and gluon amplitudes from quantum open strings in $A d S_{5} \times S^{5}$, Nucl. Phys. B 791 (2008) 93 [arXiv:0707.4254] [InSPIRE].

[24] N. Beisert, B. Eden and M. Staudacher, Transcendentality and Crossing, J. Stat. Mech. 0701 (2007) P01021 [hep-th/0610251] [INSPIRE].

[25] T. McLoughlin and R. Roiban, Spinning strings at one-loop in $A d S_{4} \times C P^{3}$, JHEP 12 (2008) 101 [arXiv:0807.3965] [INSPIRE].

[26] L.F. Alday, G. Arutyunov and D. Bykov, Semiclassical Quantization of Spinning Strings in $A d S_{4} \times C P^{3}, J H E P 11$ (2008) 089 [arXiv:0807.4400] [INSPIRE].

[27] C. Krishnan, $A d S_{4} / C F T_{3}$ at One Loop, JHEP 09 (2008) 092 [arXiv:0807.4561] [INSPIRE].

[28] T. McLoughlin, R. Roiban and A.A. Tseytlin, Quantum spinning strings in $A d S_{4} \times C P^{3}$ : Testing the Bethe Ansatz proposal, JHEP 11 (2008) 069 [arXiv:0809.4038] [INSPIRE].

[29] N. Gromov and V. Mikhaylov, Comment on the Scaling Function in $A d S_{4} \times C P^{3}$, JHEP 04 (2009) 083 [arXiv: 0807.4897] [INSPIRE].

[30] D. Astolfi, V.G.M. Puletti, G. Grignani, T. Harmark and M. Orselli, Finite-size corrections in the $\mathrm{SU}(2) \times \mathrm{SU}(2)$ sector of type IIA string theory on $A d S_{4} \times C P^{3}$,

Nucl. Phys. B 810 (2009) 150 [arXiv:0807.1527] [InSPIRE].

[31] M.A. Bandres and A.E. Lipstein, One-Loop Corrections to Type IIA String Theory in $A d S_{4} \times C P^{3}, J H E P 04(2010) 059$ [arXiv:0911.4061] [inSPIRE].

[32] M.C. Abbott, I. Aniceto and D. Bombardelli, Quantum Strings and the $A d S_{4} / C F T_{3}$ Interpolating Function, JHEP 12 (2010) 040 [arXiv:1006.2174] [INSPIRE].

[33] M.C. Abbott and P. Sundin, The Near-Flat-Space and BMN Limits for Strings in $A d S_{4} \times C P^{3}$ at One Loop, J. Phys. A 45 (2012) 025401 [arXiv:1106.0737] [inSPIRE].

[34] D. Astolfi, V.G.M. Puletti, G. Grignani, T. Harmark and M. Orselli, Finite-size corrections for quantum strings on $A d S_{4} \times C P^{3}, J H E P 05$ (2011) 128 [arXiv:1101.0004] [INSPIRE].

[35] D. Astolfi, G. Grignani, E. Ser-Giacomi and A.V. Zayakin, Strings in $A d S_{4} \times C P^{3}$ : finite size spectrum vs. Bethe Ansatz, JHEP 04 (2012) 005 [arXiv:1111.6628] [INSPIRE].

[36] C. Lopez-Arcos and H. Nastase, Eliminating ambiguities for quantum corrections to strings moving in $A d S_{4} \times C P^{3}$, Int. J. Mod. Phys. A 28 (2013) 1350058 [arXiv:1203.4777] [INSPIRE].

[37] V. Forini, V.G.M. Puletti and O. Ohlsson Sax, The generalized cusp in $A d S_{4} \times C P^{3}$ and more one-loop results from semiclassical strings, J. Phys. A 46 (2013) 115402 [arXiv: 1204.3302] [INSPIRE].

[38] I. Shenderovich, Giant magnons in $A d S_{4} / C F T_{3}$ : Dispersion, quantization and finite-size corrections, arXiv:0807.2861 [INSPIRE].

[39] B. Stefański Jr., Green-Schwarz action for Type IIA strings on $A d S_{4} \times C P^{3}$, Nucl. Phys. B 808 (2009) 80 [arXiv: 0806.4948] [INSPIRE].

[40] G. Arutyunov and S. Frolov, Superstrings on $A d S_{4} \times C P^{3}$ as a Coset $\sigma$-model, JHEP 09 (2008) 129 [arXiv:0806.4940] [INSPIRE].

[41] J. Gomis, D. Sorokin and L. Wulff, The Complete $A d S_{4} \times C P^{3}$ superspace for the type IIA superstring and D-branes, JHEP 03 (2009) 015 [arXiv:0811.1566] [INSPIRE]. 
[42] S. Giombi, R. Ricci, R. Roiban, A.A. Tseytlin and C. Vergu, Quantum $A d S_{5} \times S^{5}$ superstring in the AdS light-cone gauge, JHEP 03 (2010) 003 [arXiv:0912.5105] [INSPIRE].

[43] R.R. Metsaev and A.A. Tseytlin, Type IIB superstring action in $A d S_{5} \times S^{5}$ background, Nucl. Phys. B 533 (1998) 109 [hep-th/9805028] [INSPIRE].

[44] A. Cagnazzo, D. Sorokin and L. Wulff, String instanton in $A d S_{4} \times C P^{3}$, JHEP 05 (2010) 009 [arXiv:0911.5228] [INSPIRE].

[45] D.V. Uvarov, $A d S_{4} \times C P^{3}$ superstring in the light-cone gauge, Nucl. Phys. B 826 (2010) 294 [arXiv:0906.4699] [INSPIRE].

[46] D.V. Uvarov, Light-cone gauge Hamiltonian for $A d S_{4} \times C P^{3}$ superstring, Mod. Phys. Lett. A 25 (2010) 1251 [arXiv:0912.1044] [InSPIRE].

[47] P.A. Grassi, D. Sorokin and L. Wulff, Simplifying superstring and D-brane actions in $A d S_{4} \times C P^{3}$ superbackground, JHEP 08 (2009) 060 [arXiv:0903.5407] [INSPIRE].

[48] B. de Wit, K. Peeters, J. Plefka and A. Sevrin, The M-theory two-brane in $A d S_{4} \times S^{7}$ and $A d S_{7} \times S^{4}$, Phys. Lett. B 443 (1998) 153 [hep-th/9808052] [INSPIRE].

[49] R.R. Metsaev and A.A. Tseytlin, Superstring action in $A d S_{5} \times S^{5}$. Kappa symmetry light cone gauge, Phys. Rev. D 63 (2001) 046002 [hep-th/0007036] [inSPIRE].

[50] R.R. Metsaev, C.B. Thorn and A.A. Tseytlin, Light cone superstring in AdS space-time, Nucl. Phys. B 596 (2001) 151 [hep-th/0009171] [INSPIRE].

[51] R. Roiban, A. Tirziu and A.A. Tseytlin, Two-loop world-sheet corrections in AdS $S_{5} \times S^{5}$ superstring, JHEP 07 (2007) 056 [arXiv:0704.3638] [INSPIRE].

[52] S. Giombi, R. Ricci, R. Roiban, A.A. Tseytlin and C. Vergu, Generalized scaling function from light-cone gauge $A d S_{5} \times S^{5}$ superstring, JHEP 06 (2010) 060 [arXiv:1002.0018] [INSPIRE].

[53] S. Giombi, R. Ricci, R. Roiban and A.A. Tseytlin, Two-loop AdS $S_{5} \times S^{5}$ superstring: testing asymptotic Bethe ansatz and finite size corrections, J. Phys. A 44 (2011) 045402 [arXiv: 1010.4594] [INSPIRE].

[54] O.T. Engelund, R.W. McKeown and R. Roiban, Generalized unitarity and the worldsheet $S$ matrix in $A d S_{n} \times S^{n} \times M^{10-2 n}$, JHEP 08 (2013) 023 [arXiv: 1304.4281] [INSPIRE].

[55] L. Bianchi, V. Forini and B. Hoare, Two-dimensional S-matrices from unitarity cuts, JHEP 07 (2013) 088 [arXiv: 1304.1798] [INSPIRE].

[56] L. Bianchi and B. Hoare, $A d S_{3} \times S^{3} \times M^{4}$ string S-matrices from unitarity cuts, JHEP 08 (2014) 097 [arXiv: 1405.7947] [INSPIRE].

[57] I. Bena, J. Polchinski and R. Roiban, Hidden symmetries of the AdS $S_{5} \times S^{5}$ superstring, Phys. Rev. D 69 (2004) 046002 [hep-th/0305116] [INSPIRE].

[58] D. Sorokin and L. Wulff, Evidence for the classical integrability of the complete $A d S_{4} \times C P^{3}$ superstring, JHEP 11 (2010) 143 [arXiv:1009.3498] [INSPIRE].

[59] L. Wulff, Superisometries and integrability of superstrings, arXiv:1402.3122 [INSPIRE].

[60] M. Beccaria, G.V. Dunne, V. Forini, M. Pawellek and A.A. Tseytlin, Exact computation of one-loop correction to energy of spinning folded string in $A d S_{5} \times S^{5}$, J. Phys. A 43 (2010) 165402 [arXiv:1001.4018] [INSPIRE]. 
[61] V. Forini, Quark-antiquark potential in AdS at one loop, JHEP 11 (2010) 079 [arXiv: 1009.3939] [INSPIRE].

[62] N. Drukker and V. Forini, Generalized quark-antiquark potential at weak and strong coupling, JHEP 06 (2011) 131 [arXiv:1105.5144] [INSPIRE].

[63] R. Roiban and A.A. Tseytlin, Spinning superstrings at two loops: Strong-coupling corrections to dimensions of large-twist SYM operators, Phys. Rev. D 77 (2008) 066006 [arXiv:0712.2479] [INSPIRE].

[64] O. Bergman and S. Hirano, Anomalous radius shift in $A d S_{4} / C F T_{3}$, JHEP 07 (2009) 016 [arXiv: 0902 .1743] [INSPIRE].

[65] O. Aharony, O. Bergman and D.L. Jafferis, Fractional M2-branes, JHEP 11 (2008) 043 [arXiv: 0807.4924] [INSPIRE].

[66] N. Gromov and G. Sizov, Exact Slope and Interpolating Functions in ABJM Theory, arXiv: 1403.1894 [INSPIRE].

[67] B. Basso, An exact slope for AdS/CFT, arXiv:1109.3154 [INSPIRE].

[68] A. Kapustin, B. Willett and I. Yaakov, Exact Results for Wilson Loops in Superconformal Chern-Simons Theories with Matter, JHEP 03 (2010) 089 [arXiv:0909.4559] [INSPIRE].

[69] M. Mariño and P. Putrov, Exact Results in ABJM Theory from Topological Strings, JHEP 06 (2010) 011 [arXiv:0912.3074] [INSPIRE].

[70] N. Drukker, M. Mariño and P. Putrov, From weak to strong coupling in ABJM theory, Commun. Math. Phys. 306 (2011) 511 [arXiv:1007.3837] [InSPIRE].

[71] L. Griguolo, D. Marmiroli, G. Martelloni and D. Seminara, The generalized cusp in ABJ(M) $N=6$ Super Chern-Simons theories, JHEP 05 (2013) 113 [arXiv:1208.5766] [INSPIRE].

[72] A. Lewkowycz and J. Maldacena, Exact results for the entanglement entropy and the energy radiated by a quark, JHEP 05 (2014) 025 [arXiv:1312.5682] [INSPIRE].

[73] M.S. Bianchi, L. Griguolo, M. Leoni, S. Penati and D. Seminara, BPS Wilson loops and Bremsstrahlung function in ABJ(M): a two loop analysis, JHEP 06 (2014) 123 [arXiv: 1402.4128] [INSPIRE].

[74] D.H. Correa, J. Aguilera-Damia and G.A. Silva, Strings in $A d S_{4} \times C P^{3}$ Wilson loops in $\mathcal{N}=6$ super Chern-Simons-matter and bremsstrahlung functions, JHEP 06 (2014) 139 [arXiv: 1405.1396] [INSPIRE].

[75] K. Zarembo, Worldsheet spectrum in AdS $S_{4} / C F T_{3}$ correspondence, JHEP 04 (2009) 135 [arXiv: 0903.1747] [INSPIRE].

[76] D.V. Uvarov, $A d S_{4} \times C P^{3}$ superstring and $D=3 N=6$ superconformal symmetry, Phys. Rev. D 79 (2009) 106007 [arXiv:0811.2813] [inSPIRE].

[77] S.-J. Rey and J.-T. Yee, Macroscopic strings as heavy quarks in large- $N$ gauge theory and anti-de Sitter supergravity, Eur. Phys. J. C 22 (2001) 379 [hep-th/9803001] [INSPIRE].

[78] R. Roiban and A.A. Tseytlin, Strong-coupling expansion of cusp anomaly from quantum superstring, JHEP 11 (2007) 016 [arXiv:0709.0681] [INSPIRE].

[79] R.W. McKeown and R. Roiban, The quantum $A d S_{5} \times S^{5}$ superstring at finite coupling, arXiv: 1308.4875 [INSPIRE]. 